\title{
Non-Relativistic Twistor Theory and Newton-Cartan Geometry
}

\author{
Maciej Dunajski, James Gundry \\ Department of Applied Mathematics and Theoretical Physics, University of Cambridge, Wilberforce Road, \\ Cambridge CB3 0WA, UK. E-mail: M.Dunajski@damtp.cam.ac.uk; J.M.Gundry@damtp.cam.ac.uk
}

Received: 13 February 2015 / Accepted: 26 June 2015

Published online: 14 January 2016 - (C) The Author(s) 2016. This article is published with open access at Springerlink.com

\begin{abstract}
We develop a non-relativistic twistor theory, in which Newton-Cartan structures of Newtonian gravity correspond to complex three-manifolds with a fourparameter family of rational curves with normal bundle $\mathcal{O} \oplus \mathcal{O}(2)$. We show that the Newton-Cartan space-times are unstable under the general Kodaira deformation of the twistor complex structure. The Newton-Cartan connections can nevertheless be reconstructed from Merkulov's generalisation of the Kodaira map augmented by a choice of a holomorphic line bundle over the twistor space trivial on twistor lines. The Coriolis force may be incorporated by holomorphic vector bundles, which in general are nontrivial on twistor lines. The resulting geometries agree with non-relativistic limits of anti-self-dual gravitational instantons.
\end{abstract}

\section{Introduction}

Over the last 6 years there has been large interest in the AdS/CFT correspondences providing the gravity duals of non-relativistic gauge theories relevant in solid-state physics [15]. A non-relativistic field theory should have a dual made out of a nonrelativistic theory of gravity. The mathematical structure of such a theory-the NewtonCartan (NC) space-time-is rather baroque. It consists of a torsion-free non-metric connection $\nabla$, a parallel degenerate metric $h$ and a parallel one-form $\theta$ in the kernel of this metric $[2,8,32]$.

In this paper we shall construct a non-relativistic twistor theory for Newton-Cartan space-times. There are several equivalent definitions of twistors for flat Minkowski space, but they lead to nonequivalent pictures once a non-relativistic limit is taken. One could define twistors as spinors for the conformal group $S U(2,2)$ and then take a Wigner contraction corresponding to a limit where the speed of light becomes infinite [12]. This approach has to be abandoned in the search for an analogue of a curved twistor correspondence [25] as a general space time does not admit any conformal isometries. Thus the linear/group theoretic structures of the twistor space become irrelevant. 
We shall instead concentrate on the holomorphic geometry of the twistor space and its one-dimensional complex submanifolds. In the non-relativistic limit the normal bundle of the twistor curves corresponding to space-time points jumps from $\mathcal{O}(1) \oplus \mathcal{O}(1)$ to $\mathcal{O} \oplus \mathcal{O}(2)$, where $\mathcal{O}(n)$ is the holomorphic line bundle over $\mathbb{C P}^{1}$ with the Chern class $n$. This results in the conformal structure becoming degenerate. We shall show that the Newtonian space-times are unstable under the general Kodaira deformation of the twistor complex structure.

The Newton-Cartan connections can nevertheless be reconstructed from Merkulov's generalisation of the Kodaira map augmented by a choice of a holomorphic line bundle over the twistor space trivial on twistor lines. The Coriolis force may be incorporated by holomorphic vector bundles, which in general are non-trivial on twistor lines. The resulting geometries agree with non-relativistic limits of anti-self-dual gravitational instantons.

The paper is organised as follows: In the next Section we shall review the NewtonCartan geometry, and show that in the presence of the time-independent Coriolis force, the general Newton-Cartan connection satisfying the Einstein equations is determined by a couple of harmonic functions on $\mathbb{R}^{3}$. In Sect. 3 we shall explain how the $\mathrm{NC}$ structures arise as limits from general relativity. We shall give examples of NC limit of the Lorentzian Taub-Nut metric, anti-self-dual (ASD) gravitational waves, and GibbonsHawking gravitational instantons. The one-parameter family of anti-self-dual Ricci flat metrics

$$
g_{\epsilon}=(1+\epsilon V)\left(d x^{2}+d y^{2}+d z^{2}\right)+\frac{1}{\epsilon(1+\epsilon V)}\left(d \tau+\epsilon^{3 / 2} A\right)^{2}
$$

depending on a harmonic function $V$ and a one-form $A$ on $\mathbb{R}^{3}$ such that $\nabla V=\nabla \wedge A$ admits a Newtonian limit $\left(h=\lim _{\epsilon \rightarrow 0}\left(g^{-1}\right), \theta=d \tau, \nabla=\lim _{\epsilon \rightarrow 0} \nabla_{g_{\epsilon}}\right)$ with the unparametrised geodesic equations of $\nabla$ given by $d^{2} \mathbf{x} / d \tau^{2}=-(1 / 2) \nabla V$. In Sect. 4 we shall introduce the spinor calculus adopted to a $3+1$ splitting, leading to Newtonian spinors. In Sect. 5 we shall review the relativistic twistor theory, and proceed to construct its non-relativistic limit. This will be done by realising the relativistic twistor space as an affine line bundle over the total space of the bundle $\mathcal{O}(2) \rightarrow \mathbb{C P}^{1}$, taking a limit of the resulting patching matrix (Sect. 5.2.1) and then (Sect. 5.2.2) by directly analysing the incidence relation for a complexified Minkowski space $M_{\mathbb{C}}$ with a speed of light $c \neq 0$. We shall prove the following

Theorem 1.1. Let $P T_{c}$ be a one-parameter family of rank-two holomorphic vector bundles over $\mathbb{C P}^{1}$ determined by a patching matrix

$$
F_{c}=\left(\begin{array}{cc}
1 & -(c \lambda)^{-1} \\
0 & \lambda^{-2}
\end{array}\right), \quad \text { where } \lambda \in \mathbb{C P}^{1}
$$

(1) If $c$ is finite, then the holomorphic sections of $P T_{c} \rightarrow \mathbb{C P}^{1}$ have normal bundle $\mathcal{O}(1) \oplus \mathcal{O}(1)$. The four-dimensional moduli space $M_{\mathbb{C}}$ of these sections carries a flat conformal structure such that two points in $M_{\mathbb{C}}$ are null separated iff the corresponding sections intersect at one point in $P T_{c}$.

(2) If $c=\infty$ then the holomorphic sections of $P T_{c} \rightarrow \mathbb{C P}^{1}$ have normal bundle $\mathcal{O} \oplus \mathcal{O}(2)$. The four-dimensional moduli space $M_{\mathbb{C}}$ of these sections admits a fibration $M_{\mathbb{C}} \rightarrow \mathbb{C}$ defined by a closed one-form $\theta \in \Lambda^{1}\left(M_{\mathbb{C}}\right)$, and a contravariant metric $h$ of rank 3 , which is non-degenerate on $\operatorname{ker}(\theta)$. 
(3) For any $c \neq 0$ (finite or not) there exists an involution $\sigma: P T_{c} \rightarrow P T_{c}$ which restricts to an antipodal map on each section, and such that the $\sigma$-invariant sections form a real four-manifold $M$ with structures as in (1) and (2).

This Theorem will be established in Sect. 5.2.1. We shall explicitly construct the sections of $P T_{c}$ that will allow us to identify $c$ with the speed of light. If $c=\infty$ then $F_{\infty}=$ $\operatorname{diag}\left(1, \lambda^{-2}\right)$ and $P T_{\infty}=\mathcal{O} \oplus \mathcal{O}(2)$, but for finite $c$ there exist holomorphic splitting matrices $H$ and $\widetilde{H}$ such that $F=\widetilde{H} \operatorname{diag}\left(\lambda^{-1}, \lambda^{-1}\right) H^{-1}$ and $P T_{c}=\mathcal{O}(1) \oplus \mathcal{O}(1)$. For all non-zero $c$ there exists a holomorphic fibration $P T_{c} \rightarrow \mathcal{O}(2)$. This fibration is trivial if $c=\infty$, which gives rise to a global twistor function on $P T_{\infty}$ and consequently to a closed one-form $\theta$ on $M_{\mathbb{C}}$. The conformal freedom in $\theta$ and $h$ are both fixed by a holomorphic canonical one-form on the base of the fibration $P T_{\infty} \rightarrow \mathbb{C P}^{1}$.

In Sect. 6 we shall discuss the Kodaira deformation theory of the complex structure underlying the non-relativistic twistor space. We shall exhibit a class of deformations that do not preserve the type of the normal bundle of twistor curves, and lead to GibbonsHawking metrics. This is a consequence of the non-vanishing of the obstruction group $H^{1}\left(\mathbb{C P}^{1}, \operatorname{End}(N)\right)=\mathbb{C}$, where $N=\mathcal{O} \oplus \mathcal{O}(2)$ is the normal bundle to non-relativistic twistor lines. We shall also discuss deformations for which the twistor curves do not change their holomorphic type.

In Sect. 7 we shall use Merkulov's relative deformation theory to give a construction of Newtonian connections on $M$ with no Coriolis term. We shall establish

Theorem 1.2. There is a 1-1 correspondence between line bundles over $P T_{\infty}$ which are trivial on $\sigma$-invariant sections of $P T_{\infty} \rightarrow \mathbb{C P}^{1}$, and Newtonian connections on $M$.

It may appear that all Theorem 1.2 does is reinterpret the Penrose transform between $\sigma$-invariant sections of $H^{1}\left(P T_{\infty}, \mathcal{O}\right)$ and solutions to the Laplace equation on $\mathbb{R}^{3}$. In fact, we are saying much more than that. The Newtonian connections of Theorem 1.2 will arise as a family of morphisms

$$
N_{\mathcal{F}} \otimes\left(\odot^{2} N_{\mathcal{F}}^{*}\right) \rightarrow T M_{\mathbb{C}} \otimes \operatorname{Sym}^{2}\left(T^{*} M_{\mathbb{C}}\right)
$$

where $N_{\mathcal{F}}$ is the normal bundle to the correspondence space

$$
\mathcal{F}=\left\{(p, \xi) \in M_{\mathbb{C}} \times P T_{\infty}, \xi \in L_{p}\right\} \subset M_{\mathbb{C}} \times P T_{\infty}
$$

in the product manifold $M_{\mathbb{C}} \times P T_{\infty}$ and where $L_{p}=\mathbb{C P}^{1}$ is a rational curve in $P T_{\infty}$ corresponding to a point $p$ in the complexified Newtonian space-time $M_{\mathbb{C}}$. The contribution from the $H^{0}\left(\mathbb{C P}^{1}, \mathcal{O}(2)\right)=\mathbb{C}^{3}$ factor in (1.2) corresponds (by the Serre duality) to a zero-rest-mass field on $M_{\mathbb{C}}$ arising from a gravitational potential $V$ in the GibbonsHawking family (1.1) with $\epsilon=c^{-2}$.

In Sect. 8, rank-two vector bundles that restrict to $\mathcal{O} \oplus \mathcal{O}(2)$ on twistor curves will lead to connections with non-zero Coriolis term.

In Appendix 1 we shall exhibit and interpret a contour integral formula

$$
\psi(x, y, u)=\frac{1}{2 \pi i} \oint_{\Gamma \subset \mathbb{C P}^{1}} e^{-\frac{1}{2} m i(x-i y) \lambda} g(x+i y+\lambda u, \lambda) d \lambda,
$$

for solutions to the $2+1$ Schrodinger equation $2 m \partial_{u} \psi=i\left(\partial_{x}{ }^{2}+\partial_{y}{ }^{2}\right) \psi$. Finally in Appendix 2 we shall show how the spin connection in the Nonlinear Graviton construction arises as a Ward transform of a rank-two holomorphic vector bundle over the relativistic deformed twistor space. 


\section{Newton-Cartan Gravity}

The trajectories of test particles in Newtonian physics with a chosen universal time $t$ are integral curves $\mathbf{x}=\mathbf{x}(t)$ of the system of ODEs

$$
\frac{d^{2} \mathbf{x}}{d t^{2}}=-\nabla V,
$$

where $V: \mathbb{R}^{3} \rightarrow \mathbb{R}$ is the Newtonian potential. One normally interprets these equations as giving rise to curved paths in a fixed three-dimensional space. The geometric perspective of Cartan [2] differs in that the particle trajectories are instead regarded as geodesics of some connection in a four-dimensional space time $M$. To introduce the necessary structures consider a four-manifold $M$ equipped with a triple $(h, \theta, \nabla)$, where $h$ is a degenerate contravariant metric of signature $(0,+,+,+), \theta$ is a one-form which belongs to a kernel of $h$ (when viewed a map from $T^{*} M$ to $T M$ ), and $\nabla$ is a torsion-free affine connection on $M$ with covariant derivative $\nabla$ which keeps $h$ and $\theta$ parallel. In local coordinates

$$
h=h^{a b} \partial_{a} \otimes \partial_{b}, \quad \theta=\theta_{a} d x^{a}, \quad \text { and } \quad \nabla_{a} h^{b c}=\nabla_{a} \theta_{b}=0 .
$$

The last two conditions do not specify $\nabla$ uniquely. The most general connection satisfying (2.2) is parametrised by an otherwise irrelevant choice of time-like vector $U$ such that $\theta_{a} U^{a}=1$ and a choice of two-form $F$. The most general connection such that $U^{a} \nabla_{a} U^{b}=0, \nabla^{[a} U^{b]}=0$ is given by the Christoffel symbols

$$
\Gamma_{a b}^{c}=\frac{1}{2} h^{d c}\left(\partial_{a} h_{b d}+\partial_{b} h_{a d}-\partial_{d} h_{a b}\right)+\partial_{(a} \theta_{b)} U^{c}+\theta_{(a} F_{b) d} h^{d c},
$$

where $\partial_{a}=\partial / \partial x^{a}$, and $h_{a b}$ is a degenerate metric uniquely determined by the conditions

$$
h_{a b} U^{b}=0, \quad h_{a c} h^{b c}=\delta_{a}^{b}-\theta_{a} U^{b} .
$$

Connections of the form (2.3) are called Galilean. A Galilean connection is called Newtonian if the two-form $F$ is closed, or equivalently if the Trautman condition [32]

$$
h^{a[b} R^{c]}(d e) a=0
$$

holds. Here $R_{b c d}^{a}$ is the curvature of $\nabla$. The condition (2.4) is required for consistency with a non-relativistic limit of general relativity (see Sect. 3). Even this additional requirement allows connections which are more general than one needs to reproduce the Newtonian theory (see Sect. 2.2). For a given $(h, \theta)$ the pair $\left(U^{a}, F_{a b}\right)$ is defined up to gauge transformations

$$
U^{a} \rightarrow U^{a}+h^{a b} \Psi_{b}, \quad F \rightarrow F+d \Phi,
$$

where $\Psi=\Psi_{a} d x^{a}$ is an arbitrary one-form, and

$$
\left.\Phi=\Psi-(U\lrcorner \Psi+\frac{1}{2} h(\Psi, \Psi)\right) \theta .
$$

Thus, if $\nabla$ is a Newtonian connection (so that $d F=0$ ), then one can locally set $F=0$ and instead work with a non-trivial vector field $U$. This approach is adapted in [29].

The absence of torsion of $\nabla$, and the consistency conditions (2.2) imply the existence of a function $t: M \rightarrow \mathbb{R}$ such that $\theta=d t$. The three-dimensional distribution spanning 
the kernel of $\theta$ is integrable in the sense of the Frobenius theorem, and the manifold $M$ admits a fibration over a real line $\mathbb{R}$ called the time axis, with a coordinate $t$

$$
M \rightarrow M / \operatorname{ker}(\theta)=\mathbb{R} .
$$

Thus the space-time $M$ in the Newton-Cartan theory is a fibre bundle over a universal time axis equipped with a Riemannian metric $h$ on the fibers.

The Einstein field equations are

$$
R_{a b}=4 \pi G \rho \theta_{a} \theta_{b},
$$

where $R_{a b}$ is the Ricci tensor of $\nabla$, the function $\rho: M \rightarrow \mathbb{R}$ is the mass density and $G$ is the gravitational constant. These equations in particular imply that the metric $h$ on threedimensional spatial fibres is flat. This allows an introduction of the Galilean coordinates $x^{a}=(t, \mathbf{x})$ such that (in general indices $a, b, \ldots$ run from 0 to 3 and $i, j, \ldots$ run from 1 to 3$)$,

$$
h=\delta^{i j} \frac{\partial}{\partial x^{i}} \otimes \frac{\partial}{\partial x^{j}}, \text { and } \theta=d t .
$$

Let us spell out some physical consequences of the Newton-Cartan formalism. In general, the free falling particles follow the geodesics of $\nabla$ which however do not need to be affinely parametrised. The statement that two events are simultaneous in Newtonian physics is invariant and coordinate independent. This is a consequence of the existence of the one-form $\theta$ and the resulting fibration $M \rightarrow \mathbb{R}$. On the other hand one can not say that two events occur at the same point in 3-space but at different times. To be able to compare two non-simultaneous events one needs to make an additional choice of Galilean coordinates such that the spatial part of the Newton-Cartan connection vanishes. The existence of such coordinates is a consequence of $\nabla h=0$, and the flatness of $h$ which itself follows from the field Eq. (2.5). Moreover the distance between two events is only invariantly defined if these events are simultaneous.

2.1. Example 1: Newtonian connection. Take $h$ to be a flat metric on the spatial fibres, i. e. $h^{i j}=\delta^{i j}, \theta=d t$ and consider $\Gamma_{00}^{i}=\delta^{i j} \partial_{j} V$ with all other components of $\nabla$ equal to zero. In this case

$$
F=d t \wedge d V
$$

and the geodesics of $\nabla$ parametrised by $t$ are the integral curves of the Newton equations of motion (2.1). The coordinate transformation

$$
\mathbf{x} \longrightarrow \hat{\mathbf{x}}=R(t) \mathbf{x}+\mathbf{a}(t),
$$

where $R$ is an element of $S O(3)$ which is allowed to depend on $t$, gives rise to the 'Coriolis forces' given by the connection components $\hat{\Gamma}_{0 j}^{i}=\dot{R}_{k j} R^{i k}$. A combination of an inertial force $\ddot{\mathbf{a}}$ and a centrifugal force $\ddot{R} \mathbf{x}$ also appear as components of the connection as

$$
\hat{\Gamma}_{00}^{i}=\delta^{i j} \partial_{j} V+R_{k}^{i}\left(\ddot{R}_{l}^{k} x^{l}-\ddot{a}^{k}\right) .
$$

The form (2.1) of the geodesic equations is preserved only if $\dot{R}=0$, $\ddot{\mathbf{a}}=0$. 
2.2. Example 2: Newton-Cartan connection with the Coriolis force. The Newtonian connection of the previous subsection can be modified to a Galilean connection which includes the general space-time dependent Coriolis force $\mathbf{B}=\mathbf{B}(\mathbf{x}, t)$. In this case the closed two-form $F$ is

$$
F=-d t \wedge E+\frac{1}{2} F_{i j} d x^{i} \wedge d x^{j}, \quad F_{i j}=2 \epsilon_{i j k} B^{k},
$$

and the corresponding non-zero connection components are

$$
\Gamma_{a b}^{c}=h^{c d} \theta_{(a} F_{b) d}, \quad \Gamma_{00}^{i}=-E^{i}, \quad \Gamma_{0 j}^{i}=\frac{1}{2} F_{j}^{i}
$$

where $\theta=d t, h^{a b}$ is a degenerate metric of signature $(0,1,1,1)$ such that $h^{a b} \theta_{b}=0$ which in our case is chosen to be $\operatorname{diag}(0,1,1,1)$.

The field equations together with the Trautman condition imply $\mathbf{B}=\nabla W$, for some function $W$, and

$$
\nabla \cdot \mathbf{E}=2|\nabla W|^{2}, \quad \nabla^{2} W=0
$$

At this stage we can not assume that $\mathbf{E}=-\nabla V$. This can be either put in by hand, or derived from an asymptotic condition that $\mathbf{B} \rightarrow \mathbf{B}(t)$ at spatial infinity. This condition, together with the uniqueness theorem for the Laplace equation implies that $\mathbf{B}$ can be set to zero. This is because if $x^{i} \rightarrow R^{i}{ }_{j}(t) x+a^{i}(t)$, then $F_{i j} \rightarrow R^{k}{ }_{i} R^{l}{ }_{j} F_{k l}+R^{k}{ }_{i} \dot{R}_{k j}$, which can be set to zero by a choice of an orthogonal matrix $R(t)$. Alternatively, the closure of $F$ implies that $\mathbf{E}$ arises from a potential if $\mathbf{B}$ does not depend on $t$. If $\mathbf{E}=-\nabla V$, then

$$
\nabla^{2} W=0, \quad \nabla^{2}\left(V+W^{2}\right)=0
$$

so that the connection is determined by two harmonic functions. In Sect. 8 we shall show how two independent harmonic functions arise from holomorphic vector bundles over a Newtonian twistor space $P T_{\infty}$ which restrict to a non-trivial vector bundle $\mathcal{O} \oplus \mathcal{O}(2)$ on each twistor line.

\section{Newton-Cartan Geometry as a Limit of General Relativity}

In this section we shall show how Newton-Cartan geometry arises as a degenerate limit of General Relativity [3,19]. Let $(M, g(\epsilon))$ be a family of pseudo-Riemannian manifolds parametrised by $\epsilon>0$, and such that

$$
g^{a b}(\epsilon)=h^{a b}+\epsilon k^{a b}+O\left(\epsilon^{2}\right)
$$

where $h^{a b}$ is a contravariant tensor on $M$ of signature $(0,+,+,+)$. The following result has been established in [19].

Theorem 3.1. Let $\theta=\theta_{a} d x^{a}$ be an $\epsilon$-independent one-form such that $h^{a b} \theta_{a}=0$, normalised by $k^{a b} \theta_{a} \theta_{b}=-1$.

If the one-form $\theta$ is closed, then the Levi-Civita connection of $g(\epsilon)$ has a well defined limit $\nabla$ as $\epsilon$ goes to zero. It is a Newton-Cartan connection (2.3) with $d F=0$. 
The Newton-Cartan structure resulting from Theorem 3.1 is $(h, \nabla, \theta)$. The Ricci tensor of $g(\epsilon)$ also has a well defined limit, and choosing a perfect fluid energy-momentum tensor for the relativistic theory leads (in the limit) to the field Eq. (2.5). See [3] for details of this derivation.

Another way to proceed [11] is to define a one-parameter family of structures $\left\{h^{a b}, t_{a b}, \nabla\right\}$ such that $(t, h)$ are parallel with respect to $\nabla$ and $t_{a b} h^{b c}=-\epsilon \delta^{c}{ }_{a}$. For $\epsilon>0$ this gives pseudo-Riemannian geometry with $g_{a b}=-t_{a b} / \epsilon$, and for $\epsilon=0$ the Newton-Cartan theory with $t_{a b}=\nabla_{a} t \nabla_{b} t$, and $\theta_{a}=\nabla_{a} t$.

3.1. Examples. We shall now give three examples of Newtonian limits corresponding to particular solutions of Einstein equations. The first example is a minor modification from [11] (where some factors appear to be wrong), and corresponds to a non-zero Coriolis force and a Newton-Cartan structure of the type (2.7). The remaining two examples give limits of anti-self-dual solutions and thus correspond to analytic continuation of the Newton-Cartan theory. The resulting geodesic equations can be analytically continued to Newton's equations of motion despite the fact that ASD gravitational instantons in Riemannian signature, or ASD $p p$-waves in neutral signature do not admit Lorentzian analytic continuations. Our motivation for considering the limits of ASD solutions will become clear in Sect. 7.2 when we construct non-relativistic twistor spaces from a limiting procedure.

3.1.1. Newtonian limit of Taub-NUT Consider a one-parameter family [11] of Lorentzian Taub-NUT metrics, with the mass parameter $m$, and the NUT charge $a$

$$
g=\frac{1}{U} d r^{2}+\left(r^{2}+\epsilon a^{2}\right)\left(d \theta^{2}+\sin ^{2} \theta d \phi^{2}\right)-\frac{U}{\epsilon}(d t+2 \epsilon a \cos \theta d \phi)^{2},
$$

where

$$
U=1-\frac{2 \epsilon\left(m r+a^{2}\right)}{r^{2}+\epsilon a^{2}} .
$$

Employing the Cartesian coordinates $(x, y, z)$

$$
r=\sqrt{x^{2}+y^{2}+z^{2}}, \quad \theta=\arccos \frac{z}{\sqrt{x^{2}+y^{2}+z^{2}}}, \quad \phi=\arctan \frac{y}{x}
$$

yields the gravitational acceleration

$$
\Gamma_{00}^{i}=x^{i}\left(\frac{2 a^{2}}{r^{4}}+\frac{m}{r^{3}}\right)=\delta^{i j} \frac{\partial}{\partial x^{i}} V, \text { where } V=\left(-\frac{m}{r}-\frac{a^{2}}{r^{2}}\right),
$$

and the Coriolis force

$$
\Gamma_{0 j}{ }^{i}=\Gamma_{j 0}{ }^{i}=\epsilon_{k j}^{i} B^{k}, \text { where } B^{k}=\frac{a x^{k}}{r^{3}}=\delta^{k i} \frac{\partial}{\partial x^{i}}\left(-\frac{a}{r}\right) .
$$

Both forces arise from scalar potentials $V$ and $W=-a / r$, but only $W$ is a harmonic function. The two potentials satisfy

$$
\nabla^{2} V+2|\nabla W|^{2}=0
$$

which implies the Ricci-flat condition (2.8). 
3.1.2. Newtonian limit of gravitational instantons Let $V$ and $A$ be respectively a harmonic function, and a one-form on $\mathbb{R}^{3}$ which satisfy the Abelian monopole equation

$$
d V=* d A,
$$

where $*$ is the Hodge endomorphism of the flat metric on $\mathbb{R}^{3}$. The one-parameter family of Gibbons Hawking metrics [13]

$$
g=(1+\epsilon V)\left(d x^{2}+d y^{2}+d z^{2}\right)+\frac{1}{\epsilon(1+\epsilon V)}\left(d \tau+\epsilon^{3 / 2} A\right)^{2}
$$

has anti-self-dual Weyl tensor and is Ricci-flat. Conversely, any Riemannian ASD Ricci-flat (and so hyper-Kähler) four-manifold admitting a Killing vector preserving the hyper-Kahler structure is of the form (3.1). The Newtonian limit of (3.1) is

$$
h^{i j}=\delta^{i j}, \quad \Gamma_{\tau \tau}^{i}=\frac{1}{2} \delta^{i j} \frac{\partial V}{\partial x^{j}}, \quad \theta=d \tau,
$$

where all other components of $\Gamma_{b c}^{a}$ vanish.

For example $V=1 / r$ corresponds to the ASD Taub-NUT metric. It is an asymptotically locally flat (ALF) gravitational instanton. The metric (3.1) is everywhere regular, and asymptotically approaches the non-trivial $S^{1}$ fibration over $S^{2}$ with the monopole number 1 . In the Newtonian limit the fibration becomes trivial, so that the connection is asymptotically flat, but the genuine singularity arises at $r=0$.

3.1.3. Newtonian limit of anti-self-dual plane waves The most general ASD Ricci-flat metric admitting a parallel Killing vector is of the form [27]

$$
g=d w d x+d z d y+\gamma(w, y) d w^{2} .
$$

If the coordinates $(w, z, x, y)$ on $M$, and the arbitrary function $\gamma=\gamma(w, y)$ are taken to be real, then the metric has signature $(2,2)$. Set

$$
w=\frac{t}{\sqrt{\epsilon}}+u, \quad x=\frac{t}{\sqrt{\epsilon}}-u
$$

and redefine $\gamma$. This, for a given $\gamma$, gives a one-parameter family of metrics

$$
g=\frac{1}{\epsilon} d t^{2}-d u^{2}+d z d y+\gamma(t+\sqrt{\epsilon} u, y)(d t+\sqrt{\epsilon} d u)^{2} .
$$

with a Newtonian limit

$$
\begin{aligned}
& h \equiv \lim _{\epsilon \rightarrow 0}\left(g^{-1}\right)=-\partial_{u} \odot \partial_{u}+2 \partial_{y} \odot \partial_{z}, \quad \theta \odot \theta \equiv \lim _{\epsilon \rightarrow 0}(\epsilon g)=d t \odot d t, \\
& \Gamma_{t t}^{z}=-\frac{\partial \gamma}{\partial y},
\end{aligned}
$$

where now $\gamma=\gamma(t, y)$. This structure can, for certain $\gamma \mathrm{s}$, be analytically continued to a Newtonian theory, ${ }^{1}$ where the signature of $h$ is $(0,1,1,1)$.

${ }^{1}$ Consider a different way of taking the limit, and set $z=t / \sqrt{\epsilon}+u, y=t / \sqrt{\epsilon}-u$ so that

$$
g=\epsilon^{-1} d t^{2}+d w d x-d u^{2}+\gamma(w, t-\sqrt{\epsilon} u) d w^{2} .
$$




\section{Newtonian Spinors}

Let $(M, g)$ be a (pseudo) Riemannian four-manifold. Locally there exist complex ranktwo vector bundles $\mathbb{S}$ and $\mathbb{S}^{\prime}$ over $M$ equipped with covariantly constant symplectic structures $\varepsilon$ and $\varepsilon^{\prime}$ parallel w.r.t the Levi-Civita connection and such that

$$
\mathbb{C} \otimes T M \cong \mathbb{S} \otimes \mathbb{S}^{\prime}
$$

is a canonical bundle isomorphism, and

$$
g\left(p_{1} \otimes q_{1}, p_{2} \otimes q_{2}\right)=\varepsilon\left(p_{1}, p_{2}\right) \varepsilon^{\prime}\left(q_{1}, q_{2}\right)
$$

for $p_{1}, p_{2} \in \Gamma(\mathbb{S})$ and $q_{1}, q_{2} \in \Gamma\left(\mathbb{S}^{\prime}\right)$. We use the conventions of Penrose and Rindler [26], where the spinor indices are capital letters, unprimed for sections of $\mathbb{S}$ and primed for sections of $\mathbb{S}^{\prime}$. For example $p^{A}$ denotes a section of $\mathbb{S}$, and $q_{A^{\prime}}$ a section of $\left(\mathbb{S}^{\prime}\right)^{*}$. The symplectic structures $\varepsilon_{A B}$ and $\varepsilon_{A^{\prime} B^{\prime}}$ (such that $\varepsilon_{01}=\varepsilon_{0^{\prime} 1^{\prime}}=1$ ) are used to lower and raise the spinor indices according to $p_{A}:=p^{B} \varepsilon_{B A}, p^{A}=\varepsilon^{A B} p_{B}$. The properties of the complex conjugation on spinors depends on the signature of $g$.

- If $g$ is Lorentzian, then the spinor complex conjugation is a map $\mathbb{S} \rightarrow \mathbb{S}^{\prime}$ given by

$$
p^{A}=\left(p^{0}, p^{1}\right) \rightarrow \bar{p}^{A^{\prime}}=\left(\overline{p^{0}}, \overline{p^{1}}\right) .
$$

Linear $S L(2, \mathbb{C})$ transformations on $\mathbb{S}$ induce Lorentz transformations on vectors, and $S O(3,1)=S L(2, \mathbb{C}) / \mathbb{Z}_{2}$.

- If $g$ is Riemannian, then the complex conjugation preserves the type of spinors, i.e. it maps sections of $\mathbb{S}$ to sections of $\mathbb{S}$ and sections of $\mathbb{S}^{\prime}$ to sections of $\mathbb{S}^{\prime}$. It is given by

$$
p^{A}=\left(p^{0}, p^{1}\right) \rightarrow \hat{p}^{A}=\left(\overline{p^{1}},-\overline{p^{0}}\right), \quad q_{A^{\prime}}=\left(q_{0^{\prime}}, q_{1^{\prime}}\right) \rightarrow \hat{q}_{A^{\prime}}=\left(-\overline{q_{1^{\prime}}}, \overline{q_{0^{\prime}}}\right) .
$$

In the Riemannian case the structure group is not simple, and $S O(4, \mathbb{R})=S U(2) \times$ $S U(2)^{\prime} / \mathbb{Z}_{2}$, where the spin groups $S U(2)$ and $S U(2)^{\prime}$ act linearly on sections of $\mathbb{S}$ and $\mathbb{S}^{\prime}$ respectively.

Now assume that $g$ has Lorentzian signature, and chose a time-like unit vector $T$ to perform the $3+1$ split of $g$. This vector gives a map from $\mathbb{S}$ to $\mathbb{S}^{\prime}$ given by [28]

$$
p^{A} \rightarrow T_{A}^{A^{\prime}} p^{A},
$$

where we have chosen

$$
T^{A A^{\prime}}=\frac{1}{\sqrt{2}}\left(o^{A} o^{A^{\prime}}+\iota^{A} \iota^{A^{\prime}}\right)
$$

Footnote 1 continued

Now the limit is

$$
h=-4 \gamma \partial_{x} \odot \partial_{x}+\partial_{x} \odot \partial_{w}-\partial_{u} \odot \partial_{u}, \quad \Gamma_{w w}^{x}=\frac{\partial \gamma}{\partial w}, \quad \Gamma_{t w}^{x}=\frac{\partial \gamma}{\partial t} .
$$

This appears to be different than (3.4), but isn't, as the coordinate transformation $\hat{x}=x+4 \kappa, \hat{w}=w, u=$ $u, \hat{t}=t$ where $\gamma=\partial_{w} \kappa$ gives $h=\partial_{\hat{w}} \odot \partial_{\hat{x}}-\partial_{\hat{u}} \odot \partial_{\hat{u}}$. 
where $\left(o^{A^{\prime}}, \iota^{A^{\prime}}\right)$ is the normalised basis of $\mathbb{S}^{\prime}$, i.e. $\varepsilon_{A^{\prime} B^{\prime}} O^{A^{\prime}} \iota^{B^{\prime}}=1$, and $\left(o^{A}, \iota^{A}\right)$ is the normalised basis of $\mathbb{S}$. Under this map the isomorphism (4.1) becomes

$$
\begin{aligned}
\mathbb{C} \otimes T M & \cong \mathbb{S}^{\prime} \otimes \mathbb{S}^{\prime} \\
& =\left(\mathbb{S}^{\prime} \odot \mathbb{S}^{\prime}\right) \oplus \Lambda^{2}\left(\mathbb{S}^{\prime}\right),
\end{aligned}
$$

where $\Lambda^{2}\left(\mathbb{S}^{\prime}\right)$ is a rank-one vector bundle, and $\operatorname{Sym}^{2}\left(\mathbb{S}^{\prime}\right)$ is a rank-three vector bundle isomorphic to a bundle of self-dual two-forms on $M$.

The isomorphism (4.5) gives the orthogonal decomposition:

$$
V^{A A^{\prime}} \rightarrow V^{A A^{\prime}} T_{A}^{B^{\prime}}=V^{\left(A^{\prime} B^{\prime}\right)}+\frac{1}{2} g(V, T) \varepsilon^{A^{\prime} B^{\prime}} .
$$

The symmetric matrix $V^{\left(A^{\prime} B^{\prime}\right)}$ represents a three-vector in the space $t=$ const and the multiple of $\varepsilon^{A^{\prime} B^{\prime}}$ is the component in normal direction.

Consider the flat metric

$$
\begin{aligned}
g & =\varepsilon_{A B} \varepsilon_{A^{\prime} B^{\prime}} d x^{A A^{\prime}} \otimes d x^{B B^{\prime}} \\
& =c^{2} d t^{2}-d x^{2}-d y^{2}-d z^{2},
\end{aligned}
$$

where

$$
x^{A A^{\prime}}=\frac{1}{\sqrt{2}}\left(\begin{array}{cc}
c t+z & x+i y \\
x-i y & c t-z
\end{array}\right)
$$

(so that $T^{a} \partial / \partial x^{a}=c^{-1} \partial / \partial t$ ). The map (4.3) gives

$$
x^{A A^{\prime}} T_{A}^{B^{\prime}}=x^{A^{\prime} B^{\prime}}-\frac{1}{2} \varepsilon^{A^{\prime} B^{\prime}} c t,
$$

where

$$
x^{A^{\prime} B^{\prime}}=\frac{1}{2}\left(\begin{array}{cc}
x-i y & -z \\
-z & -x-i y
\end{array}\right) .
$$

In our discussion of the Newtonian limit of general relativity in Sect. 3 we needed to single out a time-like one-form $\theta_{a}=\nabla_{a} t$ in the kernel of the three-metric $h$. We shall use the dual vector-field to perform the $3+1$ decomposition (4.6), and construct the isomorphism (4.3). Therefore the two-component spinor formalism for Newton-Cartan structures involves only one type of spinors (which we choose to be sections of $\mathbb{S}^{\prime}$ ).

It is natural to ask whether the Newton-Cartan connection on $T M$ is induced from some spin connection on $\mathbb{S}^{\prime} \rightarrow M$. The answer to this question appears to be 'no' which is related to the fact that the Galilean boosts do not have a double cover. A NewtonCartan connection takes its values in the Lie algebra of the Galilean group. However we find that the homogeneous Galilean transformations

$$
(\mathbf{x}, t) \rightarrow(R \mathbf{x}+\mathbf{v} t, t)
$$

can not be realised spinorially as

$$
Y_{B^{\prime}}^{A^{\prime}} \rightarrow M_{C^{\prime}}^{A^{\prime}} Y^{C^{\prime}}{ }_{D^{\prime}} N^{D^{\prime}}{ }_{B^{\prime}}
$$

where $Y^{A^{\prime}}{ }_{B^{\prime}}=x^{A A^{\prime}} T_{A B^{\prime}}$ or $Y=Y_{0}+c t \mathbf{I}$, and $Y_{0}$ is trace-less. The condition (4.10) forces $M=N^{-1}$ which then implies $\mathbf{v}=0$. The choice of the unit vector (4.4) breaks 
the Lorentz invariance. A subgroup of $S L(2, \mathbb{C})$ which acts on $\mathbb{S}$ and $\mathbb{S}^{\prime}$, and preserves (4.4) is $S U$ (2). Its action on spinors only generates rotations, and not Lorentz boosts. The more general transformations of the form $Y \rightarrow M Y N+P Y$ do not work either.

It is nevertheless possible to construct a spin connection in the Newtonian limit, although to achieve a non-zero result the speed of light has to be regarded as a part of the constant metric on the fibres of $T^{*} M$ in the Cartan formalism. To see it consider the post-Newtonian limit of GR given by

$$
g=-c^{2} e^{2 V / c^{2}} d t^{2}+\delta_{i j} d x^{i} d x^{j} .
$$

Employing the spin frame $\left(c e^{V / c^{2}} d t, d x^{i}\right)$ yields a spin connection which vanishes in the limit $c \rightarrow \infty$. Let us instead consider

$$
\eta_{a b}=\left(\begin{array}{cccc}
-c^{2} & 0 & 0 & 0 \\
0 & 1 & 0 & 0 \\
0 & 0 & 1 & 0 \\
0 & 0 & 0 & 1
\end{array}\right), \quad e^{0}=e^{V / c^{2}} d t, \quad e^{i}=d x^{i}
$$

so that (4.11) is given by $g=\eta_{a b} e^{a} e^{b}$. Then the Cartan equations $d e^{a}+\omega^{a}{ }_{b} \wedge e^{b}=0$ together with a limit $c \rightarrow \infty$ yield the non-vanishing connection given by $\omega^{i}{ }_{0}=$ $\left(\partial^{i} V\right) \theta$.

\section{Twistor Theory}

5.1. Review of relativistic twistor theory. In this section we shall recall basic facts about twistor theory, following $[4,26]$.

The theory was put forward by Roger Penrose [23] in the late 1960s. The primary motivation was to unify general relativity and quantum theory in a non-local theory based on complex numbers, but a good starting point for our discussion is Penrose's contour integral formula for wave equation in Minkowski space [10,24]

$$
\phi(x, y, z, t)=\frac{1}{2 \pi i} \oint_{\Gamma \subset \mathbb{C P}^{1}} f((x+i y)+(c t+z) \lambda,(c t-z)+(x-i y) \lambda, \lambda) d \lambda,
$$

where $\Gamma \subset \mathbb{C P}^{1}$ is a closed contour and the function $f$ is holomorphic on an intersection of two open sets in $\mathbb{C P}^{1}$ away from poles inside $\Gamma$. Differentiating inside the integral shows that

$$
\frac{1}{c^{2}} \frac{\partial^{2} \phi}{\partial t^{2}}-\frac{\partial^{2} \phi}{\partial x^{2}}-\frac{\partial^{2} \phi}{\partial y^{2}}-\frac{\partial^{2} \phi}{\partial z^{2}}=0 .
$$

The formula (5.1) gives real solutions to the wave equation in Minkowski space from holomorphic functions of three variables, and the next step is to recognise these variables as coordinates in some complex three-dimensional manifold.

The five dimensional space $P N$ of light rays in the Minkowski space is a hypersurface in a three-dimensional complex manifold $P T=\mathbb{C P}^{3}-\mathbb{C P}^{1}$ called the projective twistor space. Let $\left(\omega^{0}, \omega^{1}, \pi_{0^{\prime}}, \pi_{1^{\prime}}\right) \sim \gamma\left(\omega^{0}, \omega^{1}, \pi_{0^{\prime}}, \pi_{1^{\prime}}\right), \gamma \in \mathbb{C}^{*}$ with $\left(\pi_{0^{\prime}}, \pi_{1^{\prime}}\right) \neq$ $(0,0)$ be homogeneous coordinates of a twistor. The Minkowski space and the twistor space are linked by the incidence relation

$$
\left(\begin{array}{l}
\omega^{0} \\
\omega^{1}
\end{array}\right)=\frac{1}{\sqrt{2}}\left(\begin{array}{cc}
c t+z & x+i y \\
x-i y & c t-z
\end{array}\right)\left(\begin{array}{l}
\pi_{0^{\prime}} \\
\pi_{1^{\prime}}
\end{array}\right)
$$



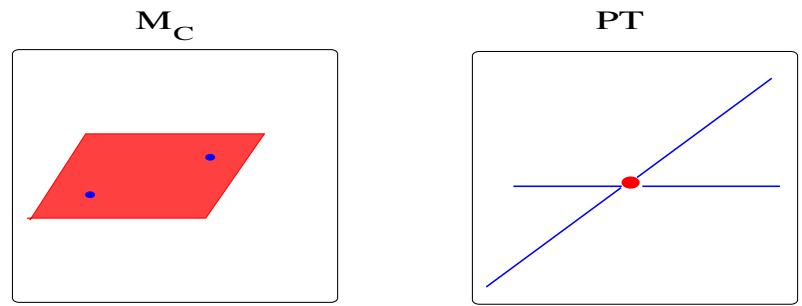

Fig. 1. Twistor relativistic incidence relation. Two points on an $\alpha$-plane in $M_{\mathbb{C}}$ correspond to two twistor lines passing through a point in $P T$

where $x^{\mu}=(t, x, y, z)$ are coordinates of a point in Minkowski space. It then follows that if two points in Minkowski space are incident with the same twistor, then they are separated by a null geodesic. The spinor form of the incidence formula above is

$$
\omega^{A}=x^{A A^{\prime}} \pi_{A^{\prime}}
$$

where the displacement vector $x^{A A^{\prime}}$ is given by (4.8).

Let $Z=\left(\omega^{A}, \pi_{A^{\prime}}\right)$ represent homogeneous coordinates of a twistor. The nonprojective twistor space $\mathbb{C}^{4}-\mathbb{C}^{2}$ is equipped with a Hermitian inner product

$$
\Sigma(Z, \bar{Z})=\omega^{A} \bar{\pi}_{A}+\bar{\omega}^{A^{\prime}} \pi_{A^{\prime}}
$$

The signature of $\Sigma$ is $(++--)$ and endomorphisms of $\mathbb{C}^{4}$ preserving $\Sigma$ as well as the orientation of $\mathbb{C}^{4}$ form a group $S U(2,2)$ which is locally isomorphic to the conformal group of the Minkowski space. The projective twistor space is divided into three regions depending on the sign of $\Sigma(Z, \bar{Z})$. The hypersurface

$$
P N=\left\{\left(\omega^{A}, \pi_{A^{\prime}}\right) \in P T, \Sigma(Z, \bar{Z})=0\right\} \subset P T
$$

is preserved by the conformal transformations of the Minkowski space.

Fixing the coordinates $(x, y, z, t)$ of a space-time point $p$ in (5.3) gives a projective line $L_{p}=\mathbb{C P}^{1}$ in $P T$. If the coordinates of $p$ are real, then $L_{p}$ is contained in the hypersurface $P N$. Conversely, fixing a point in $P N$ gives a light-ray in the Minkowski space.

Points in $P T / P N$ can be interpreted in terms of the complexified Minkowski space $M_{\mathbb{C}}=\mathbb{C}^{4}$ where they correspond to null two-dimensional planes ( $\alpha$-planes) with self-dual tangent bi-vector. This, again, is a direct consequence of (5.3) where now the coordinates $x^{A A^{\prime}}$ are complex (Fig. 1).

There is a $\mathbb{C P}^{1}$ worth of $\alpha$-planes through each point of $M_{\mathbb{C}}$. For any $\pi_{A^{\prime}} \in \mathbb{C P}^{1} \mathrm{a}$ corresponding $\alpha$-plane is spanned by a two-dimensional twistor distribution

$$
\nabla_{A}=\pi^{A^{\prime}} \partial_{A A^{\prime}}, \quad \text { where } \partial_{A A^{\prime}}=\frac{\partial}{\partial x^{A A^{\prime}}} .
$$

Finally we can give a twistor interpretation of the contour integral formula (5.1). Consider a function $f=f\left(\omega^{A} / \pi_{1^{\prime}}, \pi_{0^{\prime}} / \pi_{1^{\prime}}\right)$ which is holomorphic on an intersection of two open sets covering $P T$ (one of these sets is defined by $\pi_{1^{\prime}} \neq 0$ and the other by $\pi_{0^{\prime}} \neq 0$ ). Restrict $f$ to a rational curve $L_{p} \subset P N$ and integrate $f$ along a contour in $L_{p}$. This yields (5.1) with $\lambda=\pi_{0^{\prime}} / \pi_{1^{\prime}}$. The function $f$, when regarded as defined on the non-projective twistor space, must be homogeneous of degree -2 . 
5.2. Non-relativistic twistor theory. Our construction of a non-relativistic twistor space for the flat Newtonian space time will proceed in two steps. We shall first represent the relativistic twistor space introduced in the last section as an affine line bundle over $\mathcal{O}(2)$, and show that in the limit $c \rightarrow \infty$ the normal bundle of sections of $P T \rightarrow \mathbb{C P}^{1}$ jumps from $\mathcal{O}(1) \oplus \mathcal{O}(1)$ to $\mathcal{O} \oplus \mathcal{O}(2)$. This will be done is Sect. 5.2.1. In Sect. 5.2.2 we shall relate the jumping lines approach to a non-relativistic limit of the $3+1$ split of the twistor incidence relation.

5.2.1. Proof of Theorem 1.1 Let $\left[\pi_{0^{\prime}}, \pi_{1^{\prime}}\right]$ be homogeneous coordinates on $\mathbb{C P}^{1}$. Cover $\mathbb{C P}^{1}$ with two open sets

$$
U=\left\{[\pi] \in \mathbb{C P}^{1}, \pi_{1^{\prime}} \neq 0\right\}, \quad \widetilde{U}=\left\{[\pi] \in \mathbb{C P}^{1}, \pi_{0^{\prime}} \neq 0\right\}
$$

and set $\lambda=\pi_{0^{\prime}} / \pi_{1^{\prime}}$ on $U \cap \tilde{U}$. The Birkhoff-Grothendieck theorem states that any rank- $k$ holomorphic vector bundle over $\mathbb{C P}^{1}$ is isomorphic to a direct sum of line bundles

$$
\mathcal{O}\left(m_{1}\right) \oplus \mathcal{O}\left(m_{2}\right) \oplus \cdots \oplus \mathcal{O}\left(m_{k}\right)
$$

for some integers $m_{1}, m_{2}, \ldots, m_{k}$. Moreover the transition matrix $F: \mathbb{C}^{*} \rightarrow G L(k, \mathbb{C})$ of this bundle can be written as

$$
F=\widetilde{H} \operatorname{diag}\left(\lambda^{-m_{1}}, \lambda^{-m_{2}}, \ldots, \lambda^{-m_{k}}\right) H^{-1},
$$

where $H: U \rightarrow G L(k, \mathbb{C})$ and $\widetilde{H}: \widetilde{U} \rightarrow G L(k, \mathbb{C})$ are holomorphic.

Let $P T_{c} \longrightarrow \mathbb{C P}^{1}$ be a one-parameter family of rank-two vector bundles determined by a patching matrix

$$
F_{c}=\left(\begin{array}{cc}
1 & -(c \lambda)^{-1} \\
0 & \lambda^{-2}
\end{array}\right)
$$

where $c$ is a constant. If $c=\infty$ then $F_{\infty}=\operatorname{diag}\left(1, \lambda^{-2}\right)$ is the patching matrix for $P T_{\infty}=\mathcal{O} \oplus \mathcal{O}(2)$ with $H$ and $\widetilde{H}$ in (5.9) both equal to the identity matrix. From now on we shall identify the constant $c$ with the speed of light, and refer to $P T_{\infty}$ as the Newtonian twistor space.

If $c \neq \infty$ then

$$
F_{c}=\left(\begin{array}{cc}
0 & -c^{-1} \\
c & \lambda^{-1}
\end{array}\right)\left(\begin{array}{cc}
\lambda^{-1} & 0 \\
0 & \lambda^{-1}
\end{array}\right)\left(\begin{array}{cc}
1 & 0 \\
-c \lambda & 1
\end{array}\right)
$$

which is of the form (5.9) with

$$
\widetilde{H}=\left(\begin{array}{cc}
0 & -c^{-1} \\
c & \lambda^{-1}
\end{array}\right), \quad H=\left(\begin{array}{cc}
1 & 0 \\
-c \lambda & 1
\end{array}\right)^{-1} .
$$

Thus $P T_{c}=\mathcal{O}(1) \oplus \mathcal{O}(1)$ if the speed of light is non-zero and finite. This is the relativistic twistor space, with the holomorphic sections of $P T_{c} \rightarrow \mathbb{C P}^{1}$ parametrised by points in the complexified Minkowski space $M_{\mathbb{C}}$.

Let $x^{A A^{\prime}}$ given by (4.8) be a displacement vector of a point in $M_{\mathbb{C}}$ from the origin, and let $T^{A A^{\prime}}$ be a unit vector (4.4) with respect to the flat metric (4.7). Set

$$
\omega=T_{A} A^{\prime} \omega^{A} \pi_{A^{\prime}}=\frac{1}{\sqrt{2}}\left(\omega^{1} \pi_{0^{\prime}}-\omega^{0} \pi_{1^{\prime}}\right)
$$


and define inhomogeneous coordinates $(Q, T)$ and $(\widetilde{Q}, \widetilde{T})$ in pre-images of $U$ and $\widetilde{U}$ in $P T_{c}$ by

$$
\left(Q=2 \frac{\omega}{\pi_{1^{\prime}}}, T=\frac{\sqrt{2}}{c} \frac{\omega^{1}}{\pi_{1^{\prime}}}\right) \text { on } U
$$

and

$$
\left(\widetilde{Q}=2 \frac{\omega}{\pi_{0^{\prime}}{ }^{2}}, \widetilde{T}=\frac{\sqrt{2}}{c} \frac{\omega^{0}}{\pi_{0^{\prime}}}\right) \text { on } \widetilde{U} .
$$

On the pre-image of $U \cap \widetilde{U}$ in $P T_{c}$ we have

$$
\begin{aligned}
& \widetilde{Q}=\left(\frac{\pi_{1^{\prime}}}{\pi_{0^{\prime}}}\right)^{2} Q=\frac{1}{\lambda^{2}} Q \\
& \widetilde{T}=\frac{\sqrt{2} \omega^{0}}{c \pi_{0^{\prime}}}-\frac{\sqrt{2} \omega^{1}}{c \pi_{1^{\prime}}}+\frac{\sqrt{2} \omega^{1}}{c \pi_{1^{\prime}}}=T-\frac{2 \omega}{c \pi_{0^{\prime}} \pi_{1^{\prime}}}=T-\frac{1}{\lambda c} Q,
\end{aligned}
$$

or

$$
\left(\begin{array}{l}
\widetilde{T} \\
\widetilde{Q}
\end{array}\right)=F_{c}\left(\begin{array}{l}
T \\
Q
\end{array}\right)
$$

Note that on $P T_{\infty}$ there exists a global twistor function of weight 0 , given by $T=\widetilde{T}$.

Restricting the inhomogeneous coordinates to a twistor line $\omega^{A}=x^{A A^{\prime}} \pi_{A^{\prime}}$ gives

$$
\begin{aligned}
& Q=-(x+i y)-2 \lambda z+\lambda^{2}(x-i y), \quad T=t-\frac{1}{c}(z-\lambda(x-i y)) \quad \text { on } \\
& \widetilde{Q}=(x-i y)-2 \tilde{\lambda} z-\tilde{\lambda}^{2}(x+i y), \quad \widetilde{T}=t+\frac{1}{c}(z+\tilde{\lambda}(x+i y)) \quad \text { on } \tilde{U},
\end{aligned}
$$

where $\tilde{\lambda}=\pi_{1^{\prime}} / \pi_{0^{\prime}}$. Thus, if $c=\infty$, then $T=\widetilde{T}=t$ on the twistor lines, where $t$ is the global time coordinate on the complexified Newtonian space-time.

The algebraic geometry of holomorphic sections of $P T_{c} \rightarrow \mathbb{C P}^{1}$ determines the metric structure on $M_{\mathbb{C}}$ : two points in $M_{\mathbb{C}}$ are null separated iff the corresponding sections intersect at one point in $P T_{c}$. Infinitesimally, a vector in $T_{p} M_{\mathbb{C}}$ is null if the corresponding section of $N\left(L_{p}\right)$ vanishes at one point. This condition is equivalent to the existence of the unique solution $\lambda=\lambda_{0}$ for a simultaneous system

$$
\delta Q=0, \quad \delta T=0,
$$

where $Q$ and $T$ are given by (5.12), and the variation $\delta$ is w.r.t the moduli $(x, y, z, t)$ and $\operatorname{not} \lambda$. If $c$ is finite, then the conformal structure on $M_{\mathbb{C}}$ is determined by $\operatorname{det}\left(d x^{A A^{\prime}}\right)=0$, and the conformal factor is fixed by a two-form $d Q \wedge d T$ on the fibres of $P T_{c}$. If $c=\infty$, then the conformal structure on Minkowski space degenerates in the following way: The equation $\delta T=0$ implies that $t=$ const, and in the Newtonian limit the null separation implies simultaneity of events. We also deduce the existence of a canonical closed oneform $d T$ on $P T_{\infty}$ which yields $\theta \equiv d t$ on $M_{\mathbb{C}}$. This is the clock of Newton-Cartan theory which defines a fibration of $M_{\mathbb{C}}$ over the time axis

$$
M_{\mathbb{C}} \rightarrow M_{\mathbb{C}} / \operatorname{ker}(\theta)=\mathbb{C} .
$$


The section of $N\left(L_{p}\right)$ will have a single zero if the two roots of the remaining equation $\delta Q=0$ coincide. Evaluating the discriminant of the quadratic form leads to a degenerate conformal structure on $M_{\mathbb{C}}$, which is determined by $h=d x^{2}+d y^{2}+d z^{2}$ on threedimensional fibres of (5.13). The conformal factor is fixed by the canonical $\mathcal{O}(2)$-valued one-form $\varepsilon^{A^{\prime} B^{\prime}} \pi_{A^{\prime}} d \pi_{B^{\prime}}$ on the fibres of $P T_{\infty} \rightarrow \mathbb{C P}^{1}$ by $\left.h^{a b}=\varepsilon^{A^{\prime}\left(C^{\prime}\right.}{ }_{\varepsilon} D^{\prime}\right) B^{\prime}$. The relation between $h_{a b}$ and $h^{a b}$ is provided by a unique choice of a vector field $U$ such that $U\lrcorner \theta=1$, and $h_{a b} U^{a}=0$. This gives a degenerate contravariant metric on $T^{*} M_{\mathbb{C}}$ such that $h^{a b} \theta_{a}=0$. The discussion of the intrinsic definition of the connection is postponed to Sect. 7.

Now we shall move on to discuss the reality conditions and establish point (3) in Theorem 1.1. The Euclidean real slice of the complexified Minkowski space $M_{\mathbb{C}}$ is characterised by an involution $\sigma: P T_{c} \rightarrow P T_{c}$ given by

$$
\sigma(Q, \lambda, T)=\left(-\frac{\bar{Q}}{\bar{\lambda}^{2}},-\frac{1}{\bar{\lambda}},-\bar{T}+\frac{1}{c} \frac{\bar{Q}}{\bar{\lambda}}\right)
$$

so that $\sigma^{2}=$ Id on the projective twistor space. This involution has no fixed points, because on the non-projective twistor space it squares to minus identity, and so for any $[Z] \in P T_{c}$ there is a unique line joining $[Z]$ to $[\sigma[Z]]$. These are the real twistor curves, which correspond to points in the Euclidean slice of $M_{\mathbb{C}}$. These $\sigma$-invariant sections are characterised by

$$
\sigma(Q)=-(x+i y)+\frac{2 z}{\bar{\lambda}}+\frac{x-i y}{\bar{\lambda}^{2}}, \quad Q=-(x+i y)-2 \lambda z+\lambda^{2}(x-i y)
$$

so that $(x, y, z)$ are real. Similarly

$$
\sigma(T)=t-\frac{1}{c}\left(z+\frac{1}{\bar{\lambda}}(x-i y)\right), \quad T=t-\frac{1}{c}(z-\lambda(x-i y))
$$

so that $\bar{t}=-t$, and $t=i \tau$ is purely imaginary. Thus the real Newtonian twistor lines in $P T_{\infty}$ are

$$
Q=-(x+i y)-2 \lambda z+\lambda^{2}(x-i y), \quad \widetilde{Q}=\lambda^{-2} Q, \quad T=\widetilde{T}=i \tau
$$

where $(x, y, z, \tau)$ are real coordinates on $M$.

It is worth remarking that in the limit when $c \rightarrow \infty$ the real (i.e. $\sigma$-invariant) twistor curves lie on the hypersurface (5.6). To see it consider the inner product $\Sigma_{c}:=c^{-1} \Sigma$, where $\Sigma$ is given by (5.5). Then

$$
\Sigma_{\infty}(Z, \bar{Z})=(T+\bar{T})\left(\left|\pi_{0^{\prime}}\right|^{2}+\left|\pi_{1^{\prime}}\right|^{2}\right) .
$$

Thus the Newtonian twistor space is divided into two regions $\operatorname{Re}(T)>0$ and $\operatorname{Re}(T)<$ 0 separated by the five-dimensional space $P N_{\infty}$ of null twistors $\operatorname{Re}(T)=0$. The difference between the Lorentzian reality conditions (twistor curves contained in $P N_{c}$ ) and Riemannian reality conditions (twistor curves invariant under an anti-holomorphic involution) disappears in the Newtonian limit $c \rightarrow \infty$.

To sum up, we have exhibited a realisation of the relativistic twistor space $\mathcal{O}(1) \oplus \mathcal{O}(1)$ as an affine line bundle over the total space of the line bundle $\mathcal{O}(2) \rightarrow \mathbb{C P}^{1}$. In the limit $c=\infty$ the holomorphic structure of $P T_{c}$ changes discontinuously: The total space of $P T_{c}$, and the normal bundle of the twistor curves both jump to $\mathcal{O} \oplus \mathcal{O}(2)$. Note that this jumping is a purely holomorphic phenomenon: the bundles $P T_{c}$ are all the same for any $c$ finite or not from the topological perspective. Our findings agree with the analysis of [31] (as well as with the recent work [17]), where the jump from $\mathcal{O}(1) \oplus \mathcal{O}(1)$ to $\mathcal{O} \oplus \mathcal{O}(2)$ was interpreted as a singularity of the conformal structure. 
5.2.2. Non-relativistic incidence relation Combining the $(3+1)$ splitting (4.9) with the incidence relation (5.4) yields

$$
x^{A^{\prime} B^{\prime}} \pi_{B^{\prime}}-\frac{1}{2} c t \pi^{A^{\prime}}=\omega^{A^{\prime}}
$$

where $x^{A^{\prime} B^{\prime}}$ is given by (4.9), and $\omega^{A^{\prime}}=T_{A} A^{\prime} \omega^{A}$. This equation blows up in the limit $c \rightarrow \infty$ but the twistor function $\omega=\omega^{A} T_{A} B^{\prime} \pi_{B^{\prime}}$ remains finite. If a pair $\left(x_{0}^{A^{\prime} B^{\prime}}, t_{0}\right)$ solves the twistor Eq. (5.16), then so does

$$
x^{A^{\prime} B^{\prime}}=x_{0}^{A^{\prime} B^{\prime}}+\pi^{A^{\prime}} \rho^{B^{\prime}}+\rho^{A^{\prime}} \pi^{B^{\prime}}, \quad t=t_{0}-\frac{2}{c} \rho_{C^{\prime}} \pi^{C^{\prime}}
$$

for any constant spinor $\rho^{A^{\prime}} \in \Gamma\left(\mathbb{S}^{\prime}\right)$. The image of the two dimensional twistor distribution (5.7) tangent to $\alpha$-planes under (4.6) is

$$
T_{A^{\prime}}^{A}: \nabla_{A} \rightarrow \nabla_{A^{\prime}}=\pi^{B^{\prime}} \partial_{A^{\prime} B^{\prime}}+\frac{1}{2 c} \pi_{A^{\prime}} \frac{\partial}{\partial t} .
$$

In the Newtonian limit this gives an integrable 2-plane distribution (the mini-twistor distribution) on the constant time three-dimensional fibres of $M \rightarrow \mathbb{C}$

$$
\mathcal{D}=\operatorname{span}\left\{\partial_{x}+i \partial_{y}+\lambda \partial_{z}, \partial_{z}-\lambda\left(\partial_{x}-i \partial_{y}\right)\right\} .
$$

The non-relativistic twistor space $P T_{\infty}$ is the three-dimensional space of leaves of $\mathcal{D}$ in the correspondence space $M_{\mathbb{C}} \times \mathbb{C P}^{1}$. To specify a leaf pick a point on the time axis, and choose a two plane (a point in a mini-twistor space $\mathcal{O}(2))$ which is null with respect to the holomorphic metric $d x^{2}+d y^{2}+d z^{2}$ on the fibres of (5.13). Thus, in the Newtonian limit the $\alpha$-planes become space-like, and they lie on the fibres of (5.13) (Fig. 2).

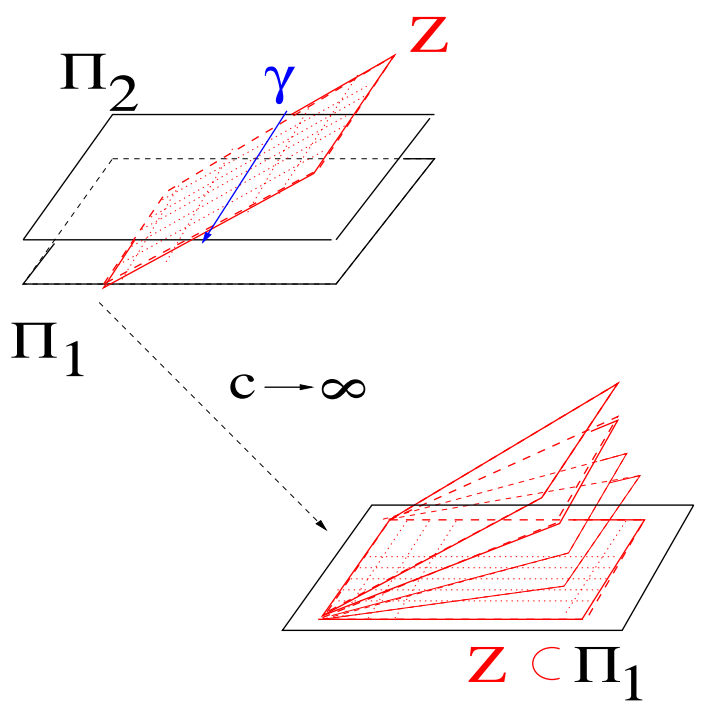

Fig. 2. An $\alpha$-plane $Z$ intersects hyper-planes $\Pi$ of constant time in $M_{\mathbb{C}}$ in null lines $\gamma$. In the non-relativistic limit $Z$ becomes a subspace of a constant-time hyper-plane $\Pi_{1}$ 


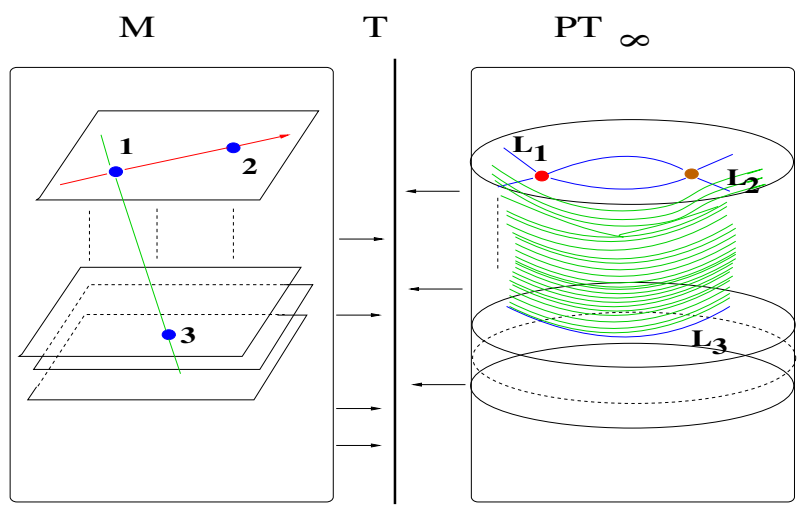

Fig. 3. A real section of the non-relativistic incidence relation. Oriented straight lines in the fibres of $M \rightarrow \mathbb{R}$ correspond to points in $P T_{\infty}$. Two simultaneous events $\mathbf{1}$ and $\mathbf{2}$ in Newtonian space-time correspond rational curves $L_{1}, L_{2}$ intersecting at two points in $P T_{\infty}$. A time-like geodesic between two non-simultaneous events 1 and $\mathbf{3}$ corresponds to a ruled surface in $P T_{\infty}$

A point $p \in M_{\mathbb{C}}$ gives a time coordinate and a $\mathbb{C P}^{1}$ worth of 2-planes on the threedimensional spatial fiber. This is a rational curve $L_{p}=\mathbb{C P}^{1}$ in $P T_{\infty}=\mathcal{O} \oplus \mathcal{O}$ (2) lying in a fibre $T=$ const. The normal bundle $N\left(L_{p}\right)$ of a rational curve $L_{p}$ corresponding to $p \in M$ is $\mathcal{O} \oplus \mathcal{O}(2)$ as we have established in Sect. 5.2.1. This can also be seen from the exact sequence

$$
0 \rightarrow \mathbb{S}^{\prime} \otimes \mathcal{O}(-1) \rightarrow \mathbb{C}^{4}=\mathbb{C}^{3} \oplus \mathbb{C} \rightarrow N\left(L_{p}\right) \rightarrow 0
$$

as the last map is, in the spinor notation, given by

$$
\left(V^{\left(A^{\prime} B^{\prime}\right)}, V^{A^{\prime} B^{\prime}} \varepsilon_{A^{\prime} B^{\prime}}\right) \rightarrow\left(V^{A^{\prime} B^{\prime}} \pi_{A^{\prime}} \pi_{B^{\prime}}, V^{A^{\prime} B^{\prime}} \varepsilon_{A^{\prime} B^{\prime}}\right)
$$

clearly projecting onto $\mathcal{O}(2) \oplus \mathcal{O}$, and the decomposition of $\mathbb{C}^{4}$ into $\mathbb{C}^{3} \oplus \mathbb{C}$ is given by (4.6).

This is a complexified picture. If the reality conditions (4.2) and (5.15) are imposed, then the intersection of a complex $\alpha$-plane with a real $\tau=$ const slice is a real straight

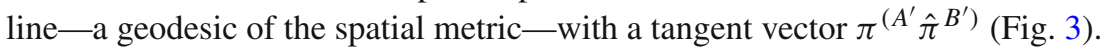

The twistor curves invariant under the involution (5.14) correspond to points in the flat Newtonian space-time $M=\mathbb{R}^{4}$. The points in $P T_{\infty}$ correspond to oriented straight lines on the fibres of $M \rightarrow \mathbb{R}$. Thus two real curves $L_{1}$ and $L_{2}$ in $P T_{\infty}$ either do not intersect, or intersect at two points $Z$ and $\sigma(Z)$ which correspond to a straight line (with two possible orientations) joining $p_{1}$ and $p_{2}$ in $M$.

\section{Kodaira Deformations}

A way to introduce the curvature on $M_{\mathbb{C}}$ in relativistic twistor theory is to deform the complex structure of $P T_{c}$. The Kodaira theorems [18] guarantee that, under the additional assumption of the deformation being 'small', the deformed twistor space will still admit a four-dimensional moduli space $M_{\mathbb{C}}$ of rational curves with normal bundle $\mathcal{O}(1) \oplus \mathcal{O}(1)$. The Nonlinear Graviton theorem of Penrose [25] then implies that $M_{\mathbb{C}}$ admits a holomorphic conformal structure with anti-self-dual (ASD) Weyl tensor, and moreover that all ASD conformal structures correspond to some deformed twistor 
spaces. If the deformation preserves the fibration of $P T_{c}$ over $\mathbb{C P}^{1}$, and the symplectic two-form $\varepsilon_{A B} d \omega^{A} \wedge d \omega^{B}$ on the fibres of this fibration, then the resulting conformal structure contains a Ricci-flat metric.

In this section we shall show that the Kodaira deformation theory, when applied to a Newtonian twistor space $P T_{\infty}$, in general leads to the jumping lines phenomenon: the deformed twistor curves have normal bundle $\mathcal{O}(1) \oplus \mathcal{O}(1)$, and thus $M_{\mathbb{C}}$ carries a nondegenerate metric, rather than a Newton-Cartan structure. We can phrase it by saying that the Newtonian space-times are unstable under the general Kodaira deformations, but we should note that this instability is a purely holomorphic feature of the underlying twistor space, and not a dynamical process.

6.1. Jumping lines and Gibbons-Hawking metrics. To consider a sub-class of deformations we shall use our construction (Sect. 5.2.1) of the undeformed Newtonian twistor space $P T_{\infty}$ as a total space of a trivial holomorphic line bundle over a total space of $\mathcal{O}(2) \rightarrow \mathbb{C P}^{1}$

$$
\mathcal{O} \oplus \mathcal{O}(2)=\mathbb{C} \times \mathcal{O}(2)
$$

where sections of $P T_{\infty} \rightarrow \mathcal{O}(2)$ restricted to sections of $\mathcal{O}(2) \rightarrow \mathbb{C P}^{1}$ are twistor curves. This motivates a replacing the undeformed twistor space $P T_{\infty}$ by

$$
L \rightarrow \mathcal{O}(2)
$$

where $L$ is a non-trivial line bundle becoming trivial on each twistor line. To construct $L$ as a deformation of $\mathcal{O} \oplus \mathcal{O}(2)$ consider the deformed patching relations

$$
\widetilde{T}=T+\epsilon f,
$$

where $f \in H^{1}\left(\mathbb{C P}^{1}, \mathcal{O}(2)\right)$ represents the cohomology class, and $\epsilon$ is a deformation parameter. Restrict this cohomology class to a section of $P T_{\infty} \rightarrow \mathbb{C P}^{1}$ and pull back to the correspondence space so that $f=\tilde{h}-h$, where $h$ and $\tilde{h}$ are holomorphic in the open sets $U$ and $\widetilde{U}$ defined by (5.8). Therefore

$$
T-\epsilon h=\widetilde{T}-\epsilon \tilde{h}=t,
$$

where $t$ does not depend on $\pi$ by the Liouville theorem and thus can be used as a coordinate on the moduli space of curves in $L$. The deformed $\mathbb{C P}^{1} \mathrm{~s}$ (in a patch containing $\lambda=0)$ are

$$
\omega(\pi)=\pi_{A^{\prime}} \pi_{B^{\prime}} x^{A^{\prime} B^{\prime}}, \quad T=t+\epsilon h,
$$

where

$$
\begin{aligned}
& h=\frac{1}{2 \pi i} \oint_{\Gamma} \frac{f(\rho, x)(\pi \cdot \iota)}{(\pi \cdot \rho)(\iota \cdot \rho)} \rho \cdot d \rho, \\
& \tilde{h}=\frac{1}{2 \pi i} \oint_{\widetilde{\Gamma}} \frac{f(\rho, x)(\pi \cdot \iota)}{(\pi \cdot \rho)(\iota \cdot \rho)} \rho \cdot d \rho .
\end{aligned}
$$

Here $\iota_{A^{\prime}}$ is a spinor (a choice of which arises because of the non-uniqueness of the splitting). The contours $\Gamma$ and $\widetilde{\Gamma}$ are homologous to the equator of the twistor curve $L_{p}=\mathbb{C P}^{1}$ in an intersection of two open sets covering $L_{p}$, and such that $\Gamma-\widetilde{\Gamma}$ surrounds the point $\rho_{A^{\prime}}=\pi_{A^{\prime}}$ in $L_{p}$ (Fig. 4). 


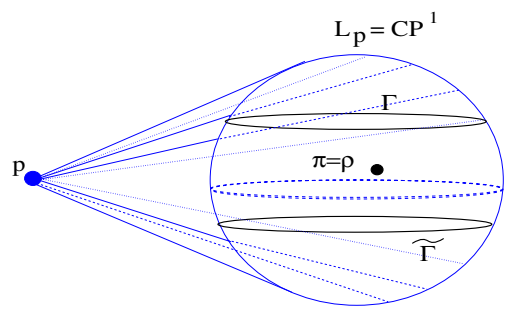

Fig. 4. Splitting formula. The contour $(\Gamma-\widetilde{\Gamma}) \subset \mathbb{C P}^{1}$ surrounds a point $\pi_{A^{\prime}}=\rho_{A^{\prime}}$

The Ward transform [33] of $L$ gives

$$
\epsilon \nabla_{A^{\prime}} h=\Phi_{A^{\prime} B^{\prime}} \pi^{B^{\prime}},
$$

where

$$
\Phi_{A^{\prime} B^{\prime}}=\epsilon A_{A^{\prime} B^{\prime}}+\epsilon \varepsilon_{A^{\prime} B^{\prime}} V
$$

is given by

$$
\Phi_{A^{\prime} B^{\prime}}=\frac{\epsilon}{2 \pi i} \oint_{\Gamma} \frac{\iota_{A^{\prime}} \rho_{B^{\prime}}}{\iota . \rho} \frac{\partial f}{\partial \omega} \rho . d \rho
$$

and the one form $A_{A^{\prime} B^{\prime}}=\Phi_{\left(A^{\prime} B^{\prime}\right)}$ together with a function $V$ satisfy the monopole equation

$$
d V=* d A
$$

or $\nabla_{A^{\prime} B^{\prime}} V=\nabla_{\left(A^{\prime}\right.}^{C^{\prime}} A_{\left.B^{\prime}\right) C^{\prime}}$. Therefore $\nabla_{A^{\prime}}=\pi^{B^{\prime}} \partial_{A^{\prime} B^{\prime}}$ is not a twistor distribution, as it does not Lie-derive $\omega$. The deformed twistor distribution (also called a Lax pair) is

$$
\begin{aligned}
L_{A^{\prime}} & =\nabla_{A^{\prime}}-\Phi_{A^{\prime} B^{\prime}} \pi^{B^{\prime}} \frac{\partial}{\partial t} \\
& =\pi^{B^{\prime}} \mathbf{e}_{A^{\prime} B^{\prime}},
\end{aligned}
$$

where $\mathbf{e}_{A^{\prime} B^{\prime}}$ are four vector fields on $M_{\mathbb{C}}$. This is a jumping line phenomenon: the deformation changes the normal bundle to $\mathcal{O}(1) \oplus \mathcal{O}(1)$. The vector fields in the Lax pair (6.6)

$$
\mathbf{e}_{A^{\prime} B^{\prime}}=\partial_{A^{\prime} B^{\prime}}-\Phi_{A^{\prime} B^{\prime}} \partial_{t}=\left\{\partial_{A^{\prime} B^{\prime}}-\epsilon A_{\left(A^{\prime} B^{\prime}\right)} \partial_{t}, \epsilon V \partial_{t}\right\}
$$

give rise, by $\mathbf{e}_{A^{\prime} B^{\prime}} \mathbf{e}^{A^{\prime} B^{\prime}}$ to a conformally rescaled Gibbons-Hawking metric. Imposing the reality conditions $t=i \tau$, where $\tau \in \mathbb{R}$ and choosing a conformal factor $V$ gives the ASD, Ricci-flat metric

$$
g=V\left(d x^{2}+d y^{2}+d z^{2}\right)+V^{-1}\left(\epsilon^{-1} d \tau+A\right)^{2} .
$$

Therefore the deformation (6.1) does not preserve the holomorphic type of twistor curves. They jump from the Newtonian $\mathcal{O} \oplus \mathcal{O}(2)$ type to relativistic $\mathcal{O}(1) \oplus \mathcal{O}(1)$.

This was to be expected as the general deformations will not preserve the type of the Newtonian normal bundle $N=\mathcal{O} \oplus \mathcal{O}(2)$. This is because $H^{1}\left(\mathbb{C P}^{1}\right.$, $\left.\operatorname{End}(N)\right) \neq 0$. If we regard a section of $N$ as a column vector with first entry taking values in $\mathcal{O}$ and the 
second entry taking values in $\mathcal{O}(2)$, the endomorphisms of $N$ are represented by two by two matrices of the form

$$
\left(\begin{array}{ll}
\alpha & \beta \\
\gamma & \delta
\end{array}\right)
$$

where $\alpha, \delta \in H^{1}\left(\mathbb{C P}^{1}, \mathcal{O}\right), \beta \in H^{1}\left(\mathbb{C P}^{1}, \mathcal{O}(-2)\right), \gamma \in H^{1}\left(\mathbb{C P}^{1}, \mathcal{O}(2)\right)$. Thus

$$
\begin{gathered}
H^{1}\left(\mathbb{C P}^{1}, \operatorname{End}(N)\right)= \\
H^{1}\left(\mathbb{C P}^{1}, \mathcal{O}\right) \oplus H^{1}\left(\mathbb{C P}^{1}, \mathcal{O}(-2)\right) \oplus H^{1}\left(\mathbb{C P}^{1}, \mathcal{O}(2)\right) \oplus H^{1}\left(\mathbb{C P}^{1}, \mathcal{O}\right)=\mathbb{C}
\end{gathered}
$$

as the second entry in the sum does not vanish.

6.2. A twisted photon construction for Newtonian space-times. The argument above shows that general Kodaira deformations of $P T_{\infty}$ do not preserve the type of the normal bundle. We have seen that a deformation which does not preserve the one-form $d T$ certainly leads to jumping lines. We can look at the restricted class of deformations which preserve $d T$, and thus do not change the normal bundle. This requires the coefficient $\beta$ in the matrix (6.7) to vanish. One way to achieve it is to take the twistor space to be a product $\mathbb{C} \times \mathcal{Z}$, where $\mathcal{Z}$ is a two-dimensional complex manifold with a three-parameter family of curves with normal bundle $\mathcal{O}(2)$. Thus $\mathcal{Z}$ is a mini-twistor space for a general Einstein-Weyl structure in three dimensions [16], and the corresponding $M$ is a product of an Einstein-Weyl three-manifold with a line. This is not the structure we are seeking, as the metric (or in fact a conformal structure) on the three-dimensional spatial slices of $M$ will in general be curved, whereas we require it to be flat. In the remainder of this section we shall explore another possibility, and deform the relation between a projective and non-projective twistor space in a way which preserves the one-form $d T$. This is analogous to the construction of [5] which in turn was motivated by Ward's twisted photon construction [33].

The canonical bundle of $P T_{\infty}$ restricted to a twistor line is $\mathcal{O}(-4)$. The argument works for $P T_{c}$ with any non-zero $c$, as restricting the canonical bundle $\kappa \rightarrow P T_{c}$ to a line $L_{p}$ with a normal bundle $N\left(L_{p}\right)$ gives

$$
\left.\kappa\right|_{L}=T^{*} L \otimes \Lambda^{2}\left(N\left(L_{p}\right)\right)^{*}=\mathcal{O}(-4)
$$

as $T^{*} L=\mathcal{O}(-2)$.

Let us assume that the line bundle $L=\kappa^{*} \otimes \mathcal{O}(-4)$ is non-trivial on $P T_{\infty}$, but trivial when restricted to twistor lines, and deform the twistor space in a way which preserves the fibration over $\mathbb{C P}^{1}$. Therefore

$$
\tilde{\pi}_{A^{\prime}}=e^{f} \pi_{A^{\prime}}
$$

where $f=\tilde{h}-h$ is the cohomology class defining $L$ which gives rise to the abelian monopole (6.3). This cohomology class coincides with that from Sect. 6.1 and its splitting is given by (6.2). The deformed twistor distribution

$$
L_{A^{\prime}}=\nabla_{A^{\prime}}-\pi^{B^{\prime}} \Phi_{A^{\prime} B^{\prime}} \Upsilon, \quad \text { where } \Upsilon=\pi^{A^{\prime}} \frac{\partial}{\partial \pi^{A^{\prime}}}
$$


Lie-derives $e^{-h} \pi_{A^{\prime}}$. To this end we note that the $\Upsilon$ term can be removed from the Lax pair by a Mobius transformation of $\pi$ iff $\Phi$ is a gradient: If $\Phi_{A^{\prime} B^{\prime}}=\nabla_{A^{\prime} B^{\prime}} U$, then setting $\tilde{x}^{A^{\prime} B^{\prime}}=x^{A^{\prime} B^{\prime}}, \tilde{\pi}^{A^{\prime}}=(\exp U) \pi^{A^{\prime}}$ gives

$$
L_{A^{\prime}}=\nabla_{A^{\prime}}+\left(\nabla_{A^{\prime}} U\right) \Upsilon=(\exp U) \widetilde{\nabla}_{A^{\prime}}
$$

In this case the deformed twistor distribution in Frobenius integrable for any function $U$.

\section{Newtonian Connections from Merkulov's Relative Deformation Theory}

The original Non-Linear Graviton construction of Penrose [25] yields Ricci-flat ASD metrics from the holomorphic geometry of associated twistor spaces. In a far reaching generalisation of this construction Merkulov [20,21] developed a twistor correspondence between complete analytic families of complex submanifolds on a given complex manifold, and a class of torsion-free affine connections (which he called the $\Lambda$-connections) on the moduli space of these submanifolds. This has subsequently led to the celebrated solution of the holonomy problem [22].

In this section we shall adapt Merkulov's construction to the Newtonian twistor correspondence, and show that the twistor space $P T_{\infty}$ introduced earlier in this paper leads to a family of Newton-Cartan connections depending on five arbitrary functions. Two of these functions can be set to zero by fixing the overall conformal factors in the degenerate Newtonian metric $h$, and the associated clock one-form $\theta$ in the kernel of $h$. The remaining three functions correspond to the gravitational force. By analysing the non-relativistic limit of the Gibbons-Hawking twistor space we shall show that the gravitational force arises from a harmonic gravitational potential which is given by a choice of a holomorphic line-bundle over $P T_{\infty}$ trivial on twistor lines.

Let $J_{p}{ }^{k}$ be an ideal of holomorphic functions on $M_{\mathbb{C}}$ which vanish to order $k$ at $p \in M_{\mathbb{C}}$. Therefore $T_{p} M_{\mathbb{C}}=\left(J_{p} / J_{p}{ }^{2}\right)^{*}$. The second-order tangent bundle $T^{[2]} M_{\mathbb{C}}$ is defined as a union over all points in $M_{\mathbb{C}}$ of second order tangent spaces $T_{p}^{[2]} M_{\mathbb{C}} \equiv$ $\left(J_{p} / J_{p}{ }^{3}\right)^{*}$. Thus an element of $\left(T_{p}^{[2]} M_{\mathbb{C}}\right)^{*}$ consists of the first two non-zero terms of a Taylor expansion of a function vanishing at $p$, and a section of $T^{[2]} M_{\mathbb{C}}$ is a second order linear differential operator $V^{[2]}=V^{a}(x) \partial_{a}+V^{a b}(x) \partial_{a} \partial_{b}$. We also have an exact sequence

$$
0 \rightarrow T M_{\mathbb{C}} \rightarrow T^{[2]} M_{\mathbb{C}} \rightarrow \operatorname{Sym}^{2}\left(T M_{\mathbb{C}}\right) \rightarrow 0
$$

such that $V^{a} \rightarrow\left(V^{a}, 0\right)$, and $\left(V^{a}, V^{a b}\right) \rightarrow V^{a b}$. A torsion-free affine connection $\nabla$ on $T M_{\mathbb{C}}$ is then equivalent to a linear map

$$
\gamma: T^{[2]} M_{\mathbb{C}} \rightarrow T M_{\mathbb{C}}
$$

where

$$
\gamma\left(V^{[2]}\right)=\left(V^{a}+\Gamma^{a}{ }_{b c} V^{b c}\right) \partial_{a}
$$

for some functions $\Gamma^{a} b c$ which are to be identified with the Christoffel symbols of $\nabla$.

In [20,21] Merkulov proposed a twistor construction of a class of maps (7.1). In some cases (e.g. the Nonlinear Graviton construction) Merkulov's construction leads to a unique connection. We will show that in the case of the non-relativistic twistor theory 
a unique Newtonian connection in Merkulov's class is provided by a natural choice of a holomorphic line bundle over $P T_{\infty}$.

The general construction, adapted to three-dimensional complex manifolds $P T_{c}$ which fiber holomorphically over $\mathbb{C P}^{1}$, and admit a moduli space $M_{\mathbb{C}}$ of holomorphic curves can be summarised as follows. Let $\mathcal{U}$ and $\widetilde{\mathcal{U}}$ be a covering of $P T_{c}$ by two open sets which is a deformation of the covering (5.11). Thus $(Q, T, \lambda)$ are holomorphic coordinates in $\mathcal{U}$ and $(\widetilde{Q}, \widetilde{T}, \tilde{\lambda})$ are holomorphic coordinates on $\widetilde{\mathcal{U}}$. The patching relation on the overlap $\mathcal{U} \cap \widetilde{\mathcal{U}}$ is $\tilde{\lambda}=\lambda^{-1}$ and $\tilde{w}^{A}=\tilde{w}^{A}\left(w^{B}, \lambda\right)$, where $w^{A}=(T, Q)$ and $\tilde{w}^{A}=(\widetilde{T}, \tilde{Q})$.

We shall make use of the Kodaira isomorphism, which relates vector fields on $M_{\mathbb{C}}$ to global sections of the normal bundle $N_{\mathcal{F}}$ to the correspondence space

$$
\mathcal{F}=\left\{(p, \xi) \in M_{\mathbb{C}} \times P T_{c}, \xi \in L_{p}\right\} \subset M_{\mathbb{C}} \times P T_{c}
$$

in the product manifold $M_{\mathbb{C}} \times P T_{c}$. The associated double fibration

$$
M_{\mathbb{C}} \stackrel{\alpha}{\longleftarrow} \mathcal{F} \stackrel{\beta}{\longrightarrow} P T_{\infty}
$$

yields

$$
N\left(L_{p}\right)=\left.N_{\mathcal{F}}\right|_{\alpha^{-1}(p)}
$$

where $L_{p}=\beta \circ \alpha^{-1}(p)=\mathbb{C P}^{1}$ is the rational curve in $P T_{c}$ corresponding to a point in $M_{\mathbb{C}}$. The patching for this normal bundle is given by

$$
F_{B}^{A}:=\left[\frac{\partial \tilde{w}^{A}}{\partial w^{B}}\right]_{\mathcal{F}}=\left(\begin{array}{ll}
\partial_{T} \widetilde{T} & \partial_{Q} \widetilde{T} \\
\partial_{T} \widetilde{Q} & \partial_{Q} \widetilde{Q}
\end{array}\right)_{\mathcal{F}}
$$

where the subscript $\mathcal{F}$ means the pull-back to the correspondence space. To exhibit the Kodaira isomorphism take a vector field given by a differential operator $V=V^{a} \partial_{a}$ on $\mathcal{F}$, and apply it to the twistor functions $\tilde{w}^{A}\left(x^{a}, \lambda\right)$ to obtain a global section of $N_{\mathcal{F}}$ given by

$$
V\left(\tilde{w}^{A}\right)=F_{B}^{A} V\left(w^{B}\right)
$$

The map (7.1) arises by taking a section of $T^{[2]} M$ and similarly applying the associated differential operator to the twistor functions. We find

$$
\begin{array}{r}
V^{[2]}\left(\tilde{w}^{A}\right)=F_{B}^{A} V^{[2]}\left(w^{B}\right)+F_{B C}^{A} V^{a b} \partial_{a} w^{B} \partial_{b} w^{C} \\
\text { where } F_{B C}^{A}:=\left[\frac{\partial^{2} \tilde{w}^{A}}{\partial w^{B} \partial w^{C}}\right]_{\mathcal{F}} .
\end{array}
$$

The term involving $F_{B C}^{A}$ is an obstruction to (7.4) representing a global section of $N_{\mathcal{F}}$; only if $F_{B C}^{A}$ splits as

$$
F_{B C}^{A}=-\tilde{\sigma}_{E F}^{A} F_{B}^{E} F_{C}^{F}+F_{D}^{A} \sigma_{B C}^{D}
$$

for some 0-cochain $\{\sigma, \tilde{\sigma}\}$ of $N_{\mathcal{F}} \otimes\left(\odot^{2} N_{\mathcal{F}}^{*}\right)$ will we arrive at

$$
V^{[2]}\left(\tilde{w}^{A}\right)+V^{a b} \tilde{\sigma}_{E F}^{A} \partial_{a} \tilde{w}^{E} \partial_{b} \tilde{w}^{F}=F_{B}^{A} V^{[2]}\left(w^{A}\right)+V^{a b} F_{B}^{A} \sigma_{E F}^{B} \partial_{a} w^{E} \partial_{b} w^{F} .
$$

In solving the splitting problem (7.5) we have constructed a global section of $N_{\mathcal{F}}$ out of a section of $T^{[2]} M$, and via the Kodaira isomorphism we have therefore constructed 
a vector field out of a section of $T^{[2]} M$. This is the map (7.1), and we can read off the connection components from

$$
\partial_{a} \partial_{b} w^{A}+\sigma_{B C}^{A} \partial_{a} w^{B} \partial_{b} w^{C}=\Gamma_{a b}^{c} \partial_{c} w^{A} .
$$

One can check that $\Gamma_{a b}^{c}$ transforms correctly as an affine connection under a change of coordinates. iff

The splitting problem (7.5) is solvable for any patching in the isomorphism class $N_{\mathcal{F}}$

$$
H^{1}\left(\mathbb{C P}^{1}, N_{\mathcal{F}} \otimes\left(\odot^{2} N_{\mathcal{F}}^{*}\right)\right)=0
$$

The procedure is however non-unique (and hence leads to a family of connections) unless $H^{0}\left(\mathbb{C P}^{1}, N_{\mathcal{F}} \otimes\left(\odot^{2} N_{\mathcal{F}}^{*}\right)\right)$ vanishes. Otherwise an element of this cohomology group can be added to $\{\sigma, \tilde{\sigma}\}$. In the relativistic case $\left.N_{\mathcal{F}}\right|_{L_{p}}=\mathcal{O}(1) \oplus \mathcal{O}(1)$ which implies that (7.7) holds, $H^{0}\left(\mathbb{C P}^{1}, N_{\mathcal{F}} \otimes\left(\odot^{2} N_{\mathcal{F}}^{*}\right)\right)=0$, and therefore the splitting is unique.

Example. Merkulov's connection for ASD plane waves To illustrate how this procedure works in practice we will consider the simple twistor space (originally due to Sparling) described by the infinitesimal deformation of the flat relativistic twistor space $P T_{c}$ given by (A4), where $f=\left(\omega^{0}\right)^{4} /\left(4 \pi_{0^{\prime}} \pi_{1^{\prime}}\right)$. Integrating the deformation equations and introducing the inhomogeneous coordinates on the deformed twistor space by $w^{A}=$ $\omega^{A} / \pi_{1^{\prime}}$ on $\mathcal{U}$ and $\tilde{w}^{A}=\tilde{\omega}^{A} / \pi_{0^{\prime}}$ on $\widetilde{\mathcal{U}}$ yields

$$
\tilde{w}^{0}=\frac{\pi_{1^{\prime}}}{\pi_{0^{\prime}}} w^{0}, \quad \tilde{w}^{1}=\frac{\pi_{1^{\prime}}}{\pi_{0^{\prime}}} w^{1}+\epsilon\left(w^{0}\right)^{3}\left(\frac{\pi_{1^{\prime}}}{\pi_{0^{\prime}}}\right)^{2} .
$$

The holomorphic splitting of these relations, together with the Liouville theorem imply the existence of coordinates $(w, z, x, y)$ on $M_{\mathbb{C}}$ such that the twistor curves pulled back from $\mathcal{U}$ to $\mathcal{F}$ are

$$
w^{0}=w+y \lambda \quad w^{1}=z-x \lambda-\epsilon y^{3} \lambda^{2} .
$$

The ASD Ricci-flat metric resulting from the Nonlinear Graviton construction is of the form (3.3) for $\gamma=-3 \epsilon y^{2}$. We shall find the connection on $M_{\mathbb{C}}$ directly. We find

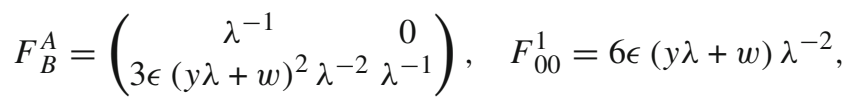

and all other $F_{B C}^{A}=0$. The splitting problem (7.5) can be solved uniquely to give

$$
\tilde{\sigma}_{00}^{1}=-6 \epsilon w, \quad \sigma_{00}^{1}=6 \epsilon y, \quad \text { and all other }\left\{\sigma_{B C}^{A}\right\}=0 \text {, }
$$

leading to a connection whose only non-vanishing components are

$$
\Gamma_{w y}^{x}=\Gamma_{y w}^{x}=-\Gamma_{w w}^{z}=-6 \epsilon y .
$$

We have recovered the Levi-Civita connection of (3.3). 
7.1. Newtonian connections from $P T_{\infty}$. We shall now construct the general class of connections on the moduli space $M_{\mathbb{C}}$ of twistor lines in $P T_{\infty}=\mathcal{O} \oplus \mathcal{O}(2)$. Using (5.10) and (7.2) we find

$$
F_{B}^{A}=\left(\begin{array}{cc}
1 & 0 \\
0 & \lambda^{-2}
\end{array}\right) \quad \text { and } \quad F_{B C}^{A}=0 .
$$

The condition $F_{B C}^{A}=0$ implies that the connections will be constructed only out of global sections of $N_{\mathcal{F}} \otimes\left(\odot^{2} N_{\mathcal{F}}^{*}\right)$. In the relativistic case this would imply $\Gamma_{a b}^{c}=0$, but for $P T_{\infty}$ we have $\left.N_{\mathcal{F}}\right|_{L_{p}}=\mathcal{O} \oplus \mathcal{O}(2)$, so

$$
\begin{aligned}
& H^{0}\left(\mathbb{C P}^{1},\left(N_{\mathcal{F}} \otimes\left(\odot^{2} N_{\mathcal{F}}^{*}\right)\right)_{L_{p}}\right) \\
& =H^{0}\left(\mathbb{C P}^{1}, \mathcal{O}(-4)\right) \oplus H^{0}\left(\mathbb{C P}^{1}, \mathcal{O}(-2)\right) \oplus H^{0}\left(\mathbb{C P}^{1}, \mathcal{O}(-2)\right) \\
& \oplus H^{0}\left(\mathbb{C P}^{1}, \mathcal{O}\right) \oplus H^{0}\left(\mathbb{C P}^{1}, \mathcal{O}\right) \oplus H^{0}\left(\mathbb{C P}^{1}, \mathcal{O}(2)\right) \\
& =\mathbb{C} \oplus \mathbb{C} \oplus \mathbb{C}^{3} .
\end{aligned}
$$

Therefore we expect $\Gamma_{a b}^{c}$ to depend on five arbitrary functions. The global sections of $N_{\mathcal{F}} \otimes\left(\odot^{2} N_{\mathcal{F}}^{*}\right)$ which constitute the Newtonian 0 -cochain $\{\sigma, \tilde{\sigma}\}_{\infty}$ are of the form

$$
\begin{aligned}
& \sigma_{T T}^{Q}=-\frac{1}{\left(\pi_{1^{\prime}}\right)^{2}} E^{A^{\prime} B^{\prime}} \pi_{A^{\prime}} \pi_{B^{\prime}}, \quad \tilde{\sigma}_{T T}^{Q}=-\frac{1}{\left(\pi_{0^{\prime}}\right)^{2}} E^{A^{\prime} B^{\prime}} \pi_{A^{\prime}} \pi_{B^{\prime}} \\
& \tilde{\sigma}_{Q T}^{Q}=\tilde{\sigma}_{T Q}^{Q}=\sigma_{Q T}^{Q}=\sigma_{T Q}^{Q}=-i \chi \quad \tilde{\sigma}_{T T}^{T}=\sigma_{T T}^{T}=-i \Sigma,
\end{aligned}
$$

(with all other components of $\left\{\sigma_{B C}^{A}\right\}_{\infty}$ set to zero) for five arbitrary functions $\left(E^{A^{\prime} B^{\prime}}, \chi, \Sigma\right)$ on $M_{\mathbb{C}}$. Using (7.10) and (7.6), and imposing the reality conditions (5.15) yields the non-vanishing connection components

$$
\Gamma_{\tau \tau}^{i}=E^{i} \quad \Gamma_{\tau \tau}^{\tau}=\Sigma \quad \Gamma_{j \tau}^{i}=\Gamma_{\tau j}^{i}=\delta_{j}^{i} \chi
$$

where we used the isomorphism (4.5) to replace $E^{A^{\prime} B^{\prime}} \in \Gamma\left(\mathbb{S}^{\prime} \odot \mathbb{S}^{\prime}\right)$ by a spatial vector $E^{i} \in \Gamma\left(T M_{\mathbb{C}}\right)$. The vector $E^{i}$ yields the gravitational attraction, whilst $\Sigma$ and $\chi$ are consistent with $\nabla h=0$ and $\nabla \theta=0$, where the degenerate metric $h$, and clock one-form $\theta$ are defined up to some conformal factors. When we fix these conformal factors as in Sect. 5.2.1, so that $h=\operatorname{diag}(0,1,1,1$,$) and \theta=d \tau$, then $\Sigma=\chi=0$. In particular, we note that this construction cannot generate a Coriolis force. What remains, then, is a connection whose non-zero $\Gamma_{\tau \tau}^{i}$ components are determined by a global section $\left\{\sigma_{T T}^{Q}\right\}_{\infty}$ of $\mathcal{O}(2)$. Using the Serre duality between the elements of $H^{1}\left(\mathbb{C P}^{1}, \mathcal{O}(-4)\right)$ and $H^{0}\left(\mathbb{C P}^{1}, \mathcal{O}(2)\right)$ we can fix this connection by

$$
E_{A^{\prime} B^{\prime}}=\frac{1}{2 \pi i} \oint_{\Gamma} g_{(-4)} \rho_{A^{\prime}} \rho_{B^{\prime}} \rho \cdot d \rho
$$

where $g_{(-4)}$ is an element of $H^{1}\left(P T_{\infty}, \mathcal{O}(-4)\right)$ restricted to a twistor line (5.12). The resulting $E_{A^{\prime} B^{\prime}}$ satisfies the zero-rest-mass field equation $\partial^{A^{\prime}}{ }_{C^{\prime}} E_{A^{\prime} B^{\prime}}=0$. In what follows we shall demonstrate that the expression (7.12) arises naturally from a limiting procedure applied to the unique splitting of the 0 -cochain corresponding to the GibbonsHawking metric. 
7.2. Proof of Theorem 1.2. In Sect. 3.1.2 we have demonstrated that any Newtonian connection with the only non-zero components given by $\Gamma_{\tau \tau}^{i}=\delta^{i j} \nabla_{j} V$ arises as a Newtonian limit of the Gibbons-Hawking metric (3.1). The twistor space of (3.1) is an affine line bundle over the total space of $\mathcal{O}(2)$ described by a cohomology class $f \in H^{1}\left(\mathbb{C P}^{1}, \mathcal{O}\right)$. This is in fact what we have recovered in our analysis in Sect. 6.1 leading to the jumping phenomenon and the Kodaira instability of the Newtonian twistor space. Let us assume that the cohomology class is represented by a function $f=f(Q, \lambda, T)$ in a patch (5.10) in $P T_{c}$, and define

$$
\widetilde{Q}=\frac{1}{\lambda^{2}} Q, \quad \widetilde{T}=T-\frac{Q}{c \lambda}-\frac{1}{c^{3}} f,
$$

with $f=0$ giving the patching relation for the undeformed relativistic twistor space $P T_{c}$ (compare 5.11). We then have

$$
\begin{aligned}
F_{B}^{A} & =\left(\begin{array}{c}
1-\left(\frac{1}{c \lambda}+\frac{1}{c^{3}}\left[\frac{\partial f}{\partial Q}\right]_{\mathcal{F}}\right) \\
\lambda^{-2}
\end{array}\right), \\
F_{A B}^{Q} & =0 \text { and } F_{A B}^{T}=\left(\begin{array}{cc}
F_{T T}^{T} & F_{T Q}^{T} \\
F_{Q T}^{T} & F_{Q Q}^{T}
\end{array}\right)_{\mathcal{F}}=\left(\begin{array}{cc}
0 & 0 \\
0-\frac{1}{c^{3}}\left[\frac{\partial^{2} f}{\partial Q^{2}}\right]_{\mathcal{F}}
\end{array}\right) .
\end{aligned}
$$

For this twistor space we have $N_{\mathcal{F}}=\mathcal{O}(1) \oplus \mathcal{O}(1)$ (for finite, non-zero $c$ ) and so one can find the unique solution $\{\sigma, \tilde{\sigma}\}_{c}$ to the splitting problem (7.5). The solution is given by

$$
\begin{aligned}
& \left(\sigma_{T T}^{Q}\right)_{c}=\frac{1}{2 \pi i} \oint_{\Gamma} \frac{1}{4\left(\pi_{1^{\prime}}\right)^{2}} \frac{\partial^{2} f}{\partial \omega^{2}}(\pi \cdot \rho)^{2} \rho \cdot d \rho+\mathcal{O}\left(\frac{1}{c}\right) \\
& \left(\tilde{\sigma}_{T T}^{Q}\right)_{c}=\left(\frac{\pi_{1^{\prime}}}{\pi_{0^{\prime}}}\right)^{2}\left(\sigma_{T T}^{Q}\right)_{c} \text { and all other }\{\sigma, \tilde{\sigma}\}_{c}=\mathcal{O}\left(\frac{1}{c}\right),
\end{aligned}
$$

where $\Gamma$ is a contour enclosing $\rho_{A^{\prime}}=0$, and $\omega=(1 / 2)\left(\rho_{1^{\prime}}\right)^{2} Q=\rho_{A^{\prime}} \rho_{B^{\prime}} x^{A^{\prime} B^{\prime}}$ is the global twistor function homogeneous of degree 2 on $P T_{\infty}$ [compare formula (5.10)] restricted to a twistor line. Thus one finds that the only parts of $\left\{\sigma_{B C}^{A}\right\}_{c}$ which do not vanish in the Newtonian limit are $\sigma_{T T}^{Q}$ and $\tilde{\sigma}_{T T}^{Q}$, constituting a global section of $\mathcal{O}(2)$ and giving rise to a non-zero $\Gamma_{\tau \tau}^{i}$ via Merkulov's procedure above. This provides a way of fixing the Newtonian 0-cochain $\sigma_{B C}^{A}$ : we identify it with the $c \rightarrow \infty$ limit of the Gibbons-Hawking 0-cochain

$$
\{\sigma, \tilde{\sigma}\}_{\infty}=\lim _{c \rightarrow \infty}\left(\{\sigma, \tilde{\sigma}\}_{c}\right)
$$

The only non-vanishing components are given by $\sigma_{T T}^{Q}$ and $\tilde{\sigma}_{T T}^{Q}$ in (7.10), where $E_{A^{\prime} B^{\prime}}$ is a zero-rest-mass field on $M_{\mathbb{C}}$. Comparing this limit with (7.12) and replacing $\partial_{A^{\prime} B^{\prime}}$ by $\rho_{A^{\prime}} \rho_{B^{\prime}} \partial / \partial \omega$ inside the integral yields

$$
\begin{aligned}
E_{A^{\prime} B^{\prime}} & =-\frac{1}{2} \frac{\partial}{\partial x^{A^{\prime} B^{\prime}}} V, \text { where } \\
V & =\frac{1}{2 \pi i} \oint_{\Gamma} \frac{1}{2} \frac{\partial f}{\partial \omega} \rho . d \rho
\end{aligned}
$$


where $f$ is an element of $H^{1}\left(P T_{\infty}, \mathcal{O}\right)$ restricted to a twistor line $\omega=x^{A^{\prime} B^{\prime}} \rho_{A^{\prime}} \rho_{B^{\prime}}$ and $V$ is the harmonic function which (up to a constant multiple) gives the Newtonian potential in agreement with (3.2).

We conclude that the limiting procedure yields a canonical element of $H^{1}\left(P T_{\infty}\right.$, $\mathcal{O}(-4))$ in (7.12). It is given by $g_{(-4)}=-(1 / 4) \partial^{2} f / \partial \omega^{2}$. To complete the proof we shall show that $f \in H^{1}\left(P T_{\infty}, \mathcal{O}\right)$ gives rise to a line bundle $v: E \rightarrow P T_{\infty}$ which is trivial on twistor curves. Let

$$
\mathcal{U}=\left\{\left(Q, T, \pi_{A^{\prime}}\right), \pi_{1^{\prime}} \neq 0\right\} \text { and } \tilde{\mathcal{U}}=\left\{\left(\tilde{Q}, T, \pi_{A^{\prime}}\right), \pi_{0^{\prime}} \neq 0\right\}
$$

be a covering of $P T_{\infty}$ and let

$$
\chi: v^{-1}(\mathcal{U}) \rightarrow \mathcal{U} \times \mathbb{C}, \quad \tilde{\chi}: v^{-1}(\widetilde{\mathcal{U}}) \rightarrow \widetilde{\mathcal{U}} \times \mathbb{C}
$$

be a local trivialisation of $E$. The holomorphic patching function $F \equiv \tilde{\chi} \circ \chi^{-1}$ is given by $F=e^{f}$. The multiplicative splitting of $F$ reduces to the additive splitting (6.2) of $f$, and it gives rise to the Abelian monopole (6.3), where $(A, V)$ satisfy (6.5). The harmonic function $V$ is identified with the Newtonian potential from Sect. 2.1. This construction gives all Newtonian connections with no Coriolis force, as in Sect. 3.1.2 we have shown that all such connections arise as limits of metrics from the Gibbons-Hawking class.

Example Consider a one-parameter family of Gibbons-Hawking metrics with linear potential [7, 14]

$$
g=(1+\epsilon z)\left(d x^{2}+d y^{2}+d z^{2}\right)+\frac{1}{\epsilon(1+\epsilon z)}\left(d \tau+\epsilon^{3 / 2} x d y\right)^{2},
$$

where $\epsilon=c^{-2}$. This family is ASD and Ricci flat for all $\epsilon>0$. In the limiting case $\epsilon \rightarrow 0$ the metric blows up, the inverse metric degenerates to $\delta^{i j}$, but the Levi-Civita connection has the finite limit with the only non-zero components given by $\Gamma_{\tau \tau}^{z}=1 / 2$. The cohomology class defining the line bundle from Theorem 1.2

$$
f=-\frac{\omega^{2}}{(\pi . o)^{2}(\pi . \iota)^{2}}
$$

gives rise to the monopole (6.4) with

$$
V=z, \quad A=\frac{1}{2}(x d y-y d x)
$$

\section{Newton-Cartan Connections from Holomorphic Vector Bundles}

Theorem 1.2 gives a satisfactory twistor construction of a Newtonian connection with no Coriolis term. For a fixed degenerate metric $h$, the general Newton-Cartan connections correspond to closed two-forms $F$, which (given a canonical 3+1 splitting induced by the one-form $\theta$ ) have well defined electric and magnetic parts (compare formulae (2.3) and (2.7)). Thus we can identify the connection with a couple of gauge fields on $M$, and construct it from a generalisation of Ward transform [33] adapted to Newtonian settings. The gauge fields needed to reconstruct the gravitational and Coriolis parts of the connection consist of a couple of abelian monopoles. The one-forms in the monopoles 
will give rise to an electric and magnetic fields on $M$, and these fields will in turn give rise to a connection.

This is a legitimate approach, as electromagnetic forces are inertial in the NewtonCartan theory: the equations of motion of a charged particle under the influence of the electric field $\mathbf{E}$ and magnetic field $2 \mathbf{B}$

$$
\ddot{\mathbf{x}}=\mathbf{E}+2 \mathbf{B} \wedge \dot{\mathbf{x}}
$$

can be reinterpreted as a geodesic motion corresponding to a connection

$$
\Gamma_{00}^{i}=-E^{i}, \quad \Gamma_{0 j}^{i}=\epsilon_{k j}^{i} B^{k},
$$

where now $\mathbf{E}$ is the gravitational acceleration, and $\mathbf{B}$ is the Coriolis force-compare (2.7). The field $\mathbf{B}$ is a gradient of a harmonic function $W$, and (assuming that $\mathbf{B}$ is timeindependent) $\mathbf{E}=-\nabla V$ is also a gradient, but $V$ is not necessarily harmonic. However $W^{2}+V$ is harmonic.

Let $M_{\mathbb{C}}$ be a moduli space of holomorphic sections of the Newtonian twistor space $P T_{\infty}=\mathcal{O} \oplus \mathcal{O}(2) \rightarrow \mathbb{C P}^{1}$, and let

$$
\mathcal{F}=\left\{(p, \xi) \in M_{\mathbb{C}} \times P T_{\infty}, \xi \in L_{p}\right\} \subset M_{\mathbb{C}} \times P T_{\infty},
$$

be the five dimensional correspondence space. Here the twistor line $L_{p}=\mathbb{C P}^{1}$ is a rational curve in $P T_{\infty}$ with normal bundle $\mathcal{O} \oplus \mathcal{O}(2)$ corresponding to $p \in M_{\mathbb{C}}$. The correspondence space can be identified with the projective spin bundle $\mathbb{P}\left(\mathbb{S}^{\prime}\right)$. Let $\mu: \mathbb{S}^{\prime} \rightarrow \mathcal{F}$ denote the corresponding fibration. Thus a pre-image of any twistor line in $\mathcal{F}$ can be pulled back to $\mathbb{S}^{\prime}$, where $\mu^{*}\left(L_{p}\right)=\mathbb{C}^{2}-\{0\}$.

Consider a vector bundle $E \rightarrow P T_{\infty}$ such that

$$
\left.E\right|_{L_{p}}=\mathcal{O}(m) \oplus \mathcal{O}(n)
$$

where $n>m \geq-1$. Therefore the restriction of $E$ to a twistor line is a non-trivial vector bundle, and the pull back to a pre-image of $L_{p}$ in $\mathcal{F}$ of the patching matrix of $E$ can not be split in a way which leads to the Ward twistor correspondence [33]. However pulling back $E$ to the total space of $\mathbb{S}^{\prime}$, and restricting it to a pre-image $\mathbb{C}^{2}-\{0\}$ of $L_{p}$ in $\mathbb{S}^{\prime}$ is a trivial bundle. The corresponding pulled-back $G L(2, \mathbb{C})$ patching matrix $F=F\left(Q, \pi_{A^{\prime}}, T\right)$, where $Q$ is given by (5.12), satisfies ${ }^{2}$

$$
F=\tilde{H} H^{-1}
$$

where $H: U \times M \rightarrow G L(2, \mathbb{C})$ and $\tilde{H}: \tilde{U} \times M \rightarrow G L(2, \mathbb{C})$ are holomorphic in $U$ and $\tilde{U}$ respectively, where now $U$ and $\tilde{U}$ are pre-images of the standard covering of $L_{p}$ in $\mathbb{C}^{2}-\{0\}$. Note that $H$ and $\tilde{H}$ are not homogeneous on $\mathbb{C}^{2}-\{0\}$ and so do not descend down to $\mathcal{F}$. Let $\nabla_{A^{\prime}}:=\pi^{B^{\prime}} \partial / \partial x^{\left(A^{\prime} B^{\prime}\right)}$ be the a rank-two twistor ( $\alpha$-plane) distribution on $\mathbb{S}^{\prime}$, such that the three-dimensional space of leaves of the rank-three distribution $\left\{\nabla_{0^{\prime}}, \nabla_{1^{\prime}}, \pi^{B^{\prime}} / \partial \pi^{B^{\prime}}\right\}$ in $\mathbb{S}^{\prime}$ is $P T_{\infty}$. Therefore $\nabla_{A^{\prime}} F=0$, and the matrix components

2 The splitting matrices $H$ and $\widetilde{H}$ can be derived from those in (5.9) using

$$
\left(\begin{array}{cc}
\left(\pi_{0^{\prime}} / \pi_{1^{\prime}}\right)^{-m} & 0 \\
0 & \left(\pi_{0^{\prime}} / \pi_{1^{\prime}}\right)^{-n}
\end{array}\right)=\left(\begin{array}{cc}
\left(\pi_{0^{\prime}}\right)^{-m} & 0 \\
0 & \left(\pi_{0^{\prime}}\right)^{-n}
\end{array}\right)\left(\begin{array}{cc}
\left(\pi_{1^{\prime}}\right)^{-m} & 0 \\
0 & \left(\pi_{1^{\prime}}\right)^{-n}
\end{array}\right)^{-1} .
$$


of $H^{-1} \nabla_{A^{\prime}} H$ are homogeneous functions on the fibres of $\mathbb{S}^{\prime}$, with the coefficients given by functions on $M$. Let us rewrite this condition as

$$
\nabla_{A^{\prime}}\left(\begin{array}{cc}
v_{-m} & r_{-n} \\
w_{-m} & s_{-n}
\end{array}\right)=\left(\begin{array}{cc}
v_{-m} & r_{-n} \\
w_{-m} & s_{-n}
\end{array}\right)\left(\begin{array}{ll}
\phi_{A^{\prime}} & \delta_{A^{\prime}} \\
\kappa_{A^{\prime}} & \psi_{A^{\prime}}
\end{array}\right), \quad \text { where } \quad H=\left(\begin{array}{cc}
v_{-m} & r_{-n} \\
w_{-m} & s_{-n}
\end{array}\right) .
$$

Here $\left(v_{-m}, r_{-n}\right)$ and $\left(w_{-m}, s_{-n}\right)$ denote a pair of local sections of $E$, where $\left(v_{-m}, w_{-m}\right)$ are homogeneous of degree $-m$ and $\left(r_{-n}, s_{-n}\right)$ are homogeneous of degree $-n$ when regarded as functions on the fibres of $\mathbb{S}^{\prime} \rightarrow M$. The polynomials $\left(\phi_{A^{\prime}}, \psi_{A^{\prime}}, \kappa_{A^{\prime}}, \delta_{A^{\prime}}\right)$ will give rise to potentials for higher spin fields on $M$, i.e. sections of various powers of $\mathbb{S}^{\prime} \rightarrow M$ satisfying some field equations [30]. To construct these fields, and find the corresponding field equations rewrite (8.2) as

$$
\begin{aligned}
\nabla_{A^{\prime}} v_{-m} & =v_{-m} \phi_{A^{\prime}}+r_{-n} \kappa_{A^{\prime}}, \\
\nabla_{A^{\prime}} w_{-m} & =w_{-m} \phi_{A^{\prime}}+s_{-n} \kappa_{A^{\prime}}, \\
\nabla_{A^{\prime}} r_{-n} & =v_{-m} \delta_{A^{\prime}}+r_{-n} \psi_{A^{\prime}}, \\
\nabla_{A^{\prime}} s_{-n} & =w_{-m} \delta_{A^{\prime}}+s_{-n} \psi_{A^{\prime}} .
\end{aligned}
$$

Therefore, as $\nabla_{A^{\prime}}$ is homogeneous of degree one, we conclude that $\phi_{A^{\prime}}$ and $\psi_{A^{\prime}}$ are homogeneous of degree one, $\kappa_{A^{\prime}}$ is homogeneous of degree $(n-m+1)$, and finally $\delta_{A^{\prime}}=0$. Equivalently

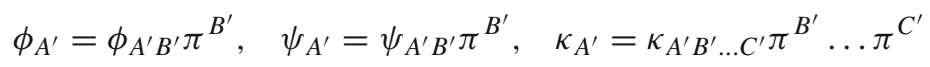

where

$\phi \in \Gamma\left(\mathbb{S}^{\prime} \otimes \mathbb{S}^{\prime}\right)=\Gamma\left(\operatorname{Sym}^{2}\left(\mathbb{S}^{\prime}\right) \oplus \mathbb{C}\right), \psi \in \Gamma\left(\mathbb{S}^{\prime} \otimes \mathbb{S}^{\prime}\right)=\Gamma\left(\operatorname{Sym}^{2}\left(\mathbb{S}^{\prime}\right) \oplus \mathbb{C}\right)$, $\kappa \in \Gamma\left(\mathbb{S}^{\prime} \otimes \operatorname{Sym}^{n-m+1}\left(\mathbb{S}^{\prime}\right)\right)=\Gamma\left(\operatorname{Sym}^{n-m+2}\left(\mathbb{S}^{\prime}\right) \oplus \operatorname{Sym}^{n-m}\left(\mathbb{S}^{\prime}\right)\right)$.

The $S L(2, \mathbb{C})$ irreducible components give rise to two spin-2 fields, two functions, one spin- $(n-m)$ field and one spin $(n-m+2)$ field.

$$
\begin{gathered}
\psi_{A^{\prime} B^{\prime}}=A_{\left(A^{\prime} B^{\prime}\right)}+\varepsilon_{A^{\prime} B^{\prime}} U, \quad \phi_{A^{\prime} B^{\prime}}=B_{\left(A^{\prime} B^{\prime}\right)}+\varepsilon_{A^{\prime} B^{\prime}} W, \\
\kappa_{A^{\prime} B^{\prime} C^{\prime} \ldots D^{\prime}}=\gamma_{\left(A^{\prime} B^{\prime} C^{\prime} \ldots D^{\prime}\right)}+\varepsilon_{A^{\prime}\left(B^{\prime}\right.} \rho_{\left.C^{\prime} \ldots D^{\prime}\right)} .
\end{gathered}
$$

To find the field equations satisfied by these potentials, we shall impose the integrability conditions on (8.3) arising from $\nabla_{A^{\prime}} \nabla^{A^{\prime}}=0$. Contracting each equation in (8.3) with $\nabla^{A^{\prime}}$ and using (8.3) to eliminate the derivatives of $H$ gives

$$
\nabla^{A^{\prime}} \phi_{A^{\prime}}=0, \quad \nabla^{A^{\prime}} \psi_{A^{\prime}}=0, \quad \nabla^{A^{\prime}} \kappa_{A^{\prime}}-\left(\phi^{A^{\prime}}-\psi^{A^{\prime}}\right) \kappa_{A^{\prime}}=0 .
$$

Decomposing this last set of equations into irreducible parts yields the final system (which is the non-relativistic version of the Sparling equations [30])

$$
\begin{aligned}
& \partial^{A^{\prime}}\left(C^{\prime} A_{\left.B^{\prime}\right) A^{\prime}}=\partial_{C^{\prime} B^{\prime}} U\right. \\
& \partial^{A^{\prime}}{ }_{\left(C^{\prime}\right.} B_{\left.B^{\prime}\right) A^{\prime}}=\partial_{C^{\prime} B^{\prime}} W
\end{aligned}
$$

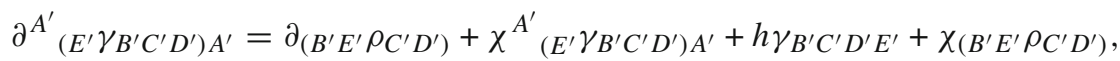


where $\chi_{A^{\prime} B^{\prime}}:=B_{A^{\prime} B^{\prime}}-A_{A^{\prime} B^{\prime}}$ and $h:=W-U$. The first two equations give a pair of Abelian monopoles $(A, U)$ and $(B, W)$ satisfying

$$
d A=* d U, \quad d B=* d W .
$$

Thus $U$ and $W$ are two harmonic functions, which give rise to electric and magnetic fields in (2.7) by

$$
\mathbf{E}=-\nabla\left(U-W^{2}\right), \quad \mathbf{B}=\nabla W
$$

If the gauge group is taken to be $S L(2, \mathbb{C})$, then $\psi_{A^{\prime} B^{\prime}}=-\phi_{A^{\prime} B^{\prime}}$.

8.0.1. A relation to Penrose-Ward correspondences Let $P T_{c}$ be a twistor space corresponding to a flat space-time with the speed of light $c$. Thus, if $c \neq \infty$, then $P T_{c}$ is the total space of a rank-two vector bundle $\mathcal{O}(1) \oplus \mathcal{O}(1)$, and $P T_{\infty}$ is the $\mathcal{O} \oplus \mathcal{O}(2)$ Newtonian twistor space.

As a special case of the construction presented in the previous section, consider a vector bundle $\mathcal{E} \rightarrow P T_{c}$, such that

$$
\left.\mathcal{E}\right|_{L_{p}}=N\left(L_{p}\right)
$$

where $N\left(L_{p}\right)$ is the holomorphic normal bundle to $L_{p} \subset P T_{c}$. Let $\kappa$ be the canonical bundle of $P T_{c}$, so that $\left.\kappa\right|_{L_{p}}=\mathcal{O}(-4)$ : this true both for finite, and infinite $c$ (as discussed in Sect. 6.2). Define a rank-two vector bundle

$$
E=\kappa^{1 / 4} \otimes \mathcal{E}
$$

If $c \neq \infty$, then $E$ is trivial when restricted to twistor lines, and its Ward correspondence gives a solution to anti-self-dual Yang-Mills field on the complexified Minkowski space. In the Newtonian case

$$
\left.E\right|_{L_{p}}=\mathcal{O}(-1) \oplus \mathcal{O}(1)
$$

corresponds to $n=1, m=-1$ in (8.2). The resulting potentials are two abelian monopoles, one spin-4 field and one spin-2 field subject to Eq. (8.4). One can now identify the field strengths of these potentials with various components of the NewtonCartan connection. Note that it is consistent to set $\gamma_{A^{\prime} B^{\prime} C^{\prime} D^{\prime}}=0$.

Acknowledgements. We are grateful to Christian Duval, George Sparling and Paul Tod for helpful discussions. This work started when M.D. was visiting the Institute for Fundamental Sciences (IMP) in Tehran in April 2010. MD is grateful to IMP for the extended hospitality when a volcanic eruption in Iceland halted air travel in Europe. The work of JG has been supported by an STFC studentship.

Open Access This article is distributed under the terms of the Creative Commons Attribution 4.0 International License (http://creativecommons.org/licenses/by/4.0/), which permits unrestricted use, distribution, and reproduction in any medium, provided you give appropriate credit to the original author(s) and the source, provide a link to the Creative Commons license, and indicate if changes were made. 


\section{Appendix 1: Contour Integral for 2 + 1 Schrödinger Equation}

It is known (see e.g. [9]) that the free Schrödinger equation in $(D, 1)$ space-time dimensions arises as a null reduction of the wave equation in $(D+1,1)$ dimensions. We shall use this observation together with the twistor contour integral formula (5.1) to construct a contour integral formula for a $(2+1)$-dimensional Schrödinger equation.

Consider the wave Eq. (5.2) which we shall write as $\square \phi=0$. Let $u=c t+z, v=c t-z$ be null coordinates, so that $\square=\partial_{x}^{2}+\partial_{y}^{2}-4 \partial_{u} \partial_{v}$. Then

$$
\phi(x, y, u, v)=e^{-\frac{i m v}{2}} \psi(x, y, u)
$$

satisfies $\square \phi=0$ iff

$$
i \frac{\partial \psi}{\partial u}=-\frac{1}{2 m}\left(\frac{\partial^{2} \psi}{\partial x^{2}}+\frac{\partial^{2} \psi}{\partial y^{2}}\right)
$$

which is the free Schrödinger equations in $2+1$ dimensions. Now consider the integral formula (5.1), where $f=f\left(\omega^{0}, \omega^{1}, \lambda\right)$ is a twistor cohomology class restricted to a twistor line (5.3). The ansatz (A1) implies that $\partial f / \partial \omega^{1}=-i m f / 2$, where $\omega^{1}=(v+\lambda(x-i y))$. Solving this equation for $f$, and substituting back into (5.1) yields the contour integral formula

$$
\psi(x, y, u)=\frac{1}{2 \pi i} \oint_{\Gamma \subset \mathbb{C P}^{1}} e^{-\frac{1}{2} m i(x-i y) \lambda} g(x+i y+\lambda u, \lambda) d \lambda,
$$

where $g$ is an element of $H^{1}(\mathcal{Z}, \mathcal{O}(-2))$ restricted to a line $L_{p} \cong \mathbb{C P}^{1}$ in $\mathcal{Z}=\mathcal{O}(1)$. The complex two fold $\mathcal{Z}$ is the twistor space of the flat holomorphic projective structure on a two-dimensional space $\mathcal{M}$. If $(\zeta, u)$ (with $\zeta=x+i y$ ) are local coordinates of a point $p \in \mathcal{M}$, then the corresponding line $L_{p} \subset \mathcal{Z}$ is given by $\eta=\zeta+\lambda u$, where $\eta$ is a coordinate on the fibres of $\mathcal{Z} \rightarrow \mathbb{C P}^{1}$. The formula (A3) is also valid if $m=0$, where the real and imaginary parts of $\psi(\zeta)$ are harmonic functions on $\mathbb{R}^{2}$ depending on a parameter $u$. In this case (A3) is the Radon transform of $g$.

Let us give an example, where $m \neq 0$. Let $g(\eta, \lambda)=\eta^{-1}$, and let $\Gamma$ be a circle centred at the origin of the $\lambda$-plane. Then

$$
\begin{aligned}
\psi & =\frac{1}{2 \pi i} \oint_{\Gamma} \frac{e^{-\frac{1}{2} m i(x-i y) \lambda}}{x+i y+\lambda u} d \lambda \\
& =\frac{1}{u} e^{\frac{i m\left(x^{2}+y^{2}\right)}{2 u}},
\end{aligned}
$$

which is indeed a solution of the $(2+1)$ Schrödinger equation (A2).

The contour integral formula (A3) is, on dimensional grounds, only tangentially related to the rest of this paper where the non-relativistic theories in $(3+1)$-dimensions are considered (hence its place in the Appendix). It may however be relevant in Newton-Cartan theories in $(2+1)$ dimensions $[1,29]$.

\section{Appendix 2: Spin Connection as Gauge Field}

In the original Nonlinear Graviton construction [25], and its modification involving non-zero cosmological constant [34] the spin connection on $\mathbb{S} \rightarrow M_{\mathbb{C}}$ can be constructed directly from the Ward correspondence applied to a certain rank-two sub-bundle of the tangent bundle of the twistor space. This construction is mentioned in [34], but not implemented explicitly. Below we show how carry the construction over in a way, which at the linearised level, agrees with the spin connection arising for Plebanski 2nd heavenly equations [27].

Let $\kappa \rightarrow P T$ be the holomorphic canonical line bundle of the twistor space which restricts to $\mathcal{O}(-4)$ on twistor lines. If $P T$ corresponds to an ASD Einstein metric (with or without $\Lambda$ ), then there exists a one-form $\mathcal{T}$ (which is given by $\pi_{A^{\prime}} d \pi^{A^{\prime}}$ in the vacuum case). This one-form defines a rank-two sub-bundle $\mathcal{E} \subset T(P T)$ consisting of vectors annihilated by $\mathcal{T}$. The bundle $\mathcal{E}$ restricts to $N$ on each twistor line. The bundle $\mathcal{E} \otimes \kappa^{1 / 4}$ is therefore trivial on twistor lines, and (by the standard Ward transform [33]) corresponds to an ASD gauge field: a spin connection on $\mathbb{S}$. This agrees with the gauge field arising from the Sparling equation in Sect. 8 . 
Both spin bundles have a twistorial construction as

$$
\mathbb{S}_{p}=\Gamma\left(L_{p}, \mathcal{E} \otimes \mathcal{O}(-1)\right), \quad \mathbb{S}_{p}^{\prime}=\Gamma\left(L_{p}, \mathcal{O}(1)\right)
$$

where $L_{p}=\mathbb{C P}^{1}$ is a twistor curve corresponding to $p \in M_{\mathbb{C}}$. Assume that the ASD metric on $M_{\mathbb{C}}$ is vacuum, and so the twistor space fibres over $\mathbb{C P}^{1}$. Let $f \in H^{1}\left(\mathbb{C} \mathbb{P}^{1}, \mathcal{O}(2)\right)$. To construction the connection on $\mathbb{S}$ consider the inifinitesimal deformation

$$
\tilde{\omega}^{A}=\omega^{A}+\epsilon \frac{\partial f}{\partial \omega_{A}}
$$

and let $U \in \Gamma(\mathcal{E})$, so that $U=U^{A A^{\prime}} \pi_{A^{\prime}} \partial / \partial \omega^{A}$. The relation

$$
\tilde{\beta}^{A} \frac{\partial}{\partial \tilde{\omega}^{A}}=\beta^{A} \frac{\partial}{\partial \omega^{A}}
$$

gives

$$
\tilde{\beta}^{B}=F_{A}^{B} \beta^{A}, \text { where } F_{A}^{B}=\delta_{A}^{B}+\epsilon \frac{\partial^{2} f}{\partial \omega_{B} \partial \omega^{A}} .
$$

To construct the splitting $F=\widetilde{H} H^{-1}$ consider

$$
\widetilde{H}=\mathbf{1}+\epsilon \widetilde{G}, \quad H=\mathbf{1}+\epsilon G
$$

so that, to the first order in $\epsilon$,

$$
\widetilde{G}_{A}^{B}-G_{A}^{B}=\frac{\partial^{2} f}{\partial \omega_{B} \partial \omega^{A}} .
$$

The freedom in splitting the RHS is measured by elements of $H^{0}\left(\mathbb{C P} \mathbb{P}^{1}, \mathcal{O}\right)$. Choosing a constant spinor $\iota_{A^{\prime}}$ yields

$$
G_{B}^{C}=\frac{1}{2 \pi i} \oint_{\Gamma} \frac{\iota . \pi}{(\iota . \rho)(\pi . \rho)} \frac{\partial^{2} f}{\partial \omega_{C} \partial \omega^{B}} \rho \cdot d \rho .
$$

The usual Liouville argument gives

$$
H^{-1} \nabla_{A} H=\widetilde{H}^{-1} \nabla_{A} \widetilde{H}=\pi^{A^{\prime}} \Gamma_{A A^{\prime}}
$$

for some matrix $\Gamma_{A A^{\prime}}$ which does not depend on $\pi_{A^{\prime}}$. Equivalently

$$
\left(H^{-1}\right)_{C}{ }^{D} \nabla_{A} H_{D}{ }^{B}=\epsilon \delta_{C}{ }^{D} \nabla_{A} G_{D}{ }^{B}=\pi^{A^{\prime}} \Gamma_{A A^{\prime} C}{ }^{B}
$$

Therefore the linearised Ward transform gives a connection

$$
\Gamma_{A A^{\prime} B}{ }^{C}=\iota_{A^{\prime}} \frac{\epsilon}{2 \pi i} \oint \frac{1}{(\iota . \rho)} \frac{\partial^{3} f}{\partial \omega_{C} \partial \omega^{B} \partial \omega^{A}} \rho . d \rho .
$$

This is consistent, in the linearised 2nd Plebanski gauge, with the expression for the ASD Weyl spinor as [6]

$$
C_{A B C}{ }^{D}=\partial_{A}{ }^{A^{\prime}} \Gamma_{B A^{\prime} C}{ }^{D}=\frac{\epsilon}{2 \pi i} \oint \frac{\partial^{4} f}{\partial \omega^{A} \partial \omega^{B} \partial \omega^{C} \partial \omega_{D}} \rho . d \rho
$$

(in Plebanski's gauge the quadratic term in the connection on $\mathbb{S}$ contracts to zero, and the connection on $\mathbb{S}^{\prime}$ vanishes). 


\section{References}

1. Andringa, R., Bergshoeff, E.A., Rosseel, J., Sezgin, E.: Newton-Cartan Supergravity; Classical Quant, Grav. 30, 205005 (2013). arXiv:1305.6737

2. Cartan, E.: Sur les varietes a connexion affine et la theorie de la relativite. Ann EC. Norm. Sup. 40, 325412 (1923)

3. Dautcourt, G.: On the Newtonian limit of general relativity. Acta. Phys. Pol. B 21, 755 (1989)

4. Dunajski, M.: Solitons, instantons \& twistors, Oxford Graduate Texts in Mathematics. Oxford University Press, Oxford (2009)

5. Dunajski, M.: The Twisted photon associated to hyper-hermitian four manifolds. J. Geom. Phys. 30, 266281 (1999)

6. Dunajski, M., Mason, L.J.: Twistor theory of hyper-Kähler metrics with hidden symmetries. J. Math. Phys. 44, 3430-3454 (2003)

7. Dunajski, M., Hoegner, M.: $S U(2)$ solutions to self-duality equations in eight dimensions. J. Geom. Phys. 62, 1747-1759 (2012)

8. Duval, C., Burdet, G., Kunzle, H.P., Perrin, M.: Bargmann structures and Newton-Cartan theory. Phys. Rev. D 31, 18411853 (1985)

9. Duval, C., Horvathy, P.A.: Non-relativistic conformal symmetries and Newton-Cartan structures. J. Phys. A 42, 465206 (2009)

10. Eastwood, M.G., Penrose, R., Wells, R.O.: Cohomology and massless fields. Commun. Math. Phys. 78, 305-351 (1980)

11. Ehlers, J.: Examples of Newtonian limits of relativistic spacetimes. Class. Quantum Grav. 14 (1997)

12. Fedoruk, S., Kosinski, P., Lukierski, J., Maslanka, P.: Nonrelativistic counterparts of twistors and the realizations of Galilean conformal algebra. Phys. Lett. B 669, 129134 (2011)

13. Gibbons, G.W., Hawking, S.W.: Gravitational multi-instantons. Phys. Lett. B 78, 430 (1978)

14. Maldonado, R., Ward, R.S.: Geometry of periodic monopoles. Phys. Rev. D 88, 125013 (2013)

15. Hartnoll, S.: Lectures on holographic methods for condensed matter physics. Class. Quant. Grav. 26, 224002 (2009)

16. Hitchin N.J.: Complex manifolds and Einstein's equations, Twistor geometry and nonlinear systems (Primorsko, 1980), Lecture Notes in Math. vol. 970, pp. 73-99. Springer, Berlin, New York (1982)

17. Hitchin, N.J.: Higgs bundles and diffeomorphism groups (2015). arXiv:1501.04989

18. Kodaira, K.: On stability of compact submanifolds of complex manifolds. Am. J. Math. 85, 79-94 (1963)

19. Künzle, H.P.: Covariant Newtonian limit of Lorentz space-times. Gen. Rel. Grav. 7, 445 (1976)

20. Merkulov, S.A.: Relative deformation theory and differential geometry. In: Twistor theory. Marcel Dekker, New York (1995)

21. Merkulov, S.A.: Geometry of Kodaira moduli spaces, Proc. Am. Math. Soc. 124, 1499-1506 (1996)

22. Merkulov, S.A., Schwachhofer, L.: Classification of irreducible holonomies of torsion-free affine connections. Ann. Math. 150, 77-149 (1999)

23. Penrose, R.: Twistor algebra. J. Math. Phys. 8, 345-366 (1967)

24. Penrose, R.: Solutions of the zero-rest-mass equations. J. Math. Phys. 10, 38 (1969)

25. Penrose, R.: Nonlinear gravitons and curved twistor theory. Gen. Rel. Grav. 7, 31-52 (1976)

26. Penrose, R., Rindler, W.: Spinors and space-time. Two-spinor calculus and relativistic fields. In: Cambridge Monographs on Mathematical Physics. Cambridge University Press, Cambridge $(1987,1988)$

27. Plebański, J.F.: Some solutions of complex Einstein equations. J. Math. Phys. 16, 2395-2402 (1975)

28. Sommers, P.: Space spinors. J. Math. Phys. 21, 2567 (1980)

29. Son, D.T.: Newton-Cartan Geometry and the Quantum Hall effect (2013). arXiv:1306.0638

30. Sparling, G.A.J.: Dynamically broken symmetry and global Yang-Mills in Minkowski space Twistor Newsletter (1977)

31. Tod, K.P.: The singularities of H-space. Math. Proc. Camb. Philos. Soc. 92, 331 (1982)

32. Trautman, A.: Sur la theorie newtonienne de la gravitation. Comptes Rendus Acad. Sci. Paris. 247, 617 (1963)

33. Ward, R.S.: On self-dual gauge fields. Phys. Lett. 61, 81-82 (1977)

34. Ward, R.S.: Self-dual space-times with cosmological constant Comm. Math. Phys. 78, 1-17 (1980) 